\title{
The Denef-Loeser series for toric surface singularities
}

\author{
Monique Lejeune-Jalabert and Ana J. Reguera
}

\begin{abstract}
Let $H$ denote the set of formal arcs going through a singular point of an algebraic variety $V$ defined over an algebraically closed field $\mathbf{k}$ of characteristic zero. In the late sixties, J. Nash has observed that for any nonnegative integer $s$, the set $j^{s}(H)$ of $s$-jets of $\operatorname{arcs}$ in $H$ is a constructible subset of some affine space. Recently (1999), J. Denef and F. Loeser have proved that the Poincaré series associated with the image of $j^{s}(H)$ in some suitable localization of the Grothendieck ring of algebraic varieties over $\mathbf{k}$ is a rational function. We compute this function for normal toric surface singularities.
\end{abstract}

\section{Introduction}

Let $V$ be an algebraic variety defined over an algebraically closed field of characteristic zero. Recently, J. Denef and F. Loeser have attached canonical rational functions to the set $H$ of formal arcs on $V$ going through a given singular point $O$ of $V$ (see [D-L1], [D-L2]). The existence of such functions is a finiteness property of the family $\left\{j^{s}(H)\right\}_{s \in \mathbb{N}}$ of $s$-jets of $\operatorname{arcs}$ in $H$, analogous to the rationality of the $p$-adic Poincaré series associated to the $p$-adic points on a variety, and it fits with Nash's expectation in $[\mathrm{N}]$.

Denef and Loeser have computed these functions for analytically irreducible plane curve singularities in [D-L2]. As far as we know, it is the only concrete example available so far. In this paper, we compute the series $P_{\text {geom }}(T)$ for toric surface singularities (the definition is given in section 2). The final formula shown in theorem 4.5 is given in terms of a natural continued fraction expansion of the quotient $q / p$ of relatively prime integers satisfying $0<p<q$, characterizing the singularity in the dictionary between toric varieties and convex cones.

2000 Mathematics Subject Classification: 14B05, 14J17, $14 \mathrm{M} 25$.

Keywords: Arc spaces, Denef-Loeser series, toric surfaces. 
Moreover, as a consequence of our proof, which only involves the blowingup with center $O$ and the normalized Nash blowing-up of the surface, we get geometric interpretations. For a toric surface singularity $(V, O), P_{\text {geom }}(T)$ has only simple poles. Except for $1-\mathbb{L}^{2} T$, which occurs for any surface germ, singular or not, these poles are in one to one correspondence with the divisorial valuations of the function field $\mathbf{k}(V)$ whose center on the normalized Nash blowing-up (resp. the blowing-up with center $O$ ) is an irreducible exceptional curve (resp. a closed point). The tangent lines whose direction is one of these points appear in [GS3] and they are named "exceptional tangents" in [L-T]. In particular, we prove that $P_{\text {geom }}(T)=\frac{1}{1-\mathbb{L}^{2} T}$ as in the nonsingular case, if and only if the toric surface $V$ has no exceptional tangent at $O$.

The paper is divided into three sections followed by an appendix. In section 2, we recall the definition of $P_{\text {geom }}(T)$ and of its ingredients: arcs, jets (or truncations) of arcs, Grothendieck ring of $\mathbf{k}$-varieties, and we state DenefLoeser's rationality theorem. The geometric analysis of the space of arcs and of its jets is carried out in section 3 . For each $s \geq 0$, we get a finite partition of the set of $s$-jets of arcs by constructible subsets isomorphic to the product of a 1 or 2-dimensional torus by an affine space of suitable dimension. These dimensions are controlled by piecewise linear functions, which turn out to be linear on the cones of the fan corresponding to the minimal modification of the surface factoring through its blowing-up with center $O$ and its Nash blowing-up. We compute the series thus obtained in section 4. Here, its rationality follows from the rationality of the series $\sum_{m \in \sigma \cap \mathbb{Z}^{2}} x^{m}$ for any convex rational polyhedral cone $\sigma$ in $\mathbb{R}^{2}$. Several characterizations of toric surface singularities with the same $P_{\text {geom }}(T)$ are given. We close this section with four questions.

The reader is expected to have some familiarity with the basic elements of the dictionary given in $[\mathrm{TE}]$ or $[\mathrm{O}$, chap. I]. We limit ourselves to recall the geometric significance of the strongly convex rational polyhedral cone classically attached to an affine normal toric variety (see (3.1)), and the description of the normalized blowing-up with respect to a monomial ideal via its Newton fan (see (3.7)). On the contrary, we have found it useful to give details on the Nash blowing-up of toric varieties. For these varieties, González-Sprinberg has exhibited an ideal whose blowing-up is a Nash blowing-up. In the appendix, we show that this ideal has a nice interpretation via differential forms with logarithmic poles. The idea of the proof and the terminology introduced originate in $[\mathrm{P}$, section 4$]$.

We denote by $V_{\sigma}$ (resp. $V_{\Sigma}$ ) the toric variety which corresponds to the cone $\sigma$ (resp. the fan $\Sigma$ ). 
Acknowledgments: We would like to thank the organizers of the International Conference on Algebraic Geometry and Singularities in honour of Professor José Luis Vicente Córdoba (Sevilla, September 2001) for their invitation. We also thank F. Loeser for his suggestions and encouragement.

\section{Arcs, jets and the series $P_{\text {geom }}(T)$}

In this preliminary section, we introduce our notation, and we recall the statement of Denef-Loeser's fundamental theorem 1.1. in [D-L1].

Let $V$ be an algebraic variety over an algebraically closed field $\mathbf{k}$, and let $O$ be a closed point on $V$. Let $D:=$ Spec $\mathbf{k}[[t]]$ and let 0 denote its closed point. A (formal) arc on $V$ going through $O$ is a k-morphism $h:(D, 0) \longrightarrow$ $(V, O)$, (or equivalently a k-local homomorphism $\left.h^{*}: \mathcal{O}_{V, O} \longrightarrow \mathbf{k}[[t]]\right)$. The set $H$ of arcs on $V$ going through $O$ is naturally endowed with a reduced scheme structure. Indeed, since the question is local and $\mathbf{k}$ is assumed to be algebraically closed, we may assume that $V$ is a closed subvariety of some affine space $\mathbb{A}_{\mathbf{k}}^{d}:=\operatorname{Spec} \mathbf{k}\left[X_{1}, \ldots, X_{d}\right]$ and that $O=(0, \ldots, 0)$. An arc $h \in H$ is given by power series expansions

$$
X_{i}(t)=\sum_{j \geq 1} x_{i, j} t^{j} \in \mathbf{k}[[t]], \quad 1 \leq i \leq d,
$$

such that $f\left(X_{1}(t), \ldots, X_{d}(t)\right) \equiv 0$ for every $f$ in the defining ideal $I(V)$ of $V$ in $\mathbb{A}_{\mathrm{k}}^{d}$. For $l \geq 1$, set $\underline{x}_{l}:=\left(x_{1, l}, \ldots, x_{d, l}\right)$ (resp. $\underline{X}_{l}:=\left(X_{1, l}, \ldots, X_{d, l}\right)$ where the $X_{i, j}$ 's are indeterminates). Observe that

$$
f\left(X_{1}(t), \ldots, X_{d}(t)\right)=\sum_{j \geq 1} f_{j}\left(\underline{x}_{1}, \ldots \underline{x}_{j}\right) t^{j}
$$

where $f_{j}$ is a quasi-homogeneous polynomial of weight $j$ in the polynomial $\operatorname{ring} \mathbf{k}\left[\underline{X}_{1}, \ldots, \underline{X}_{j}\right]$ graded by giving the weight $l$ to $X_{1, l}, \ldots, X_{d, l}$ for every $l$, $1 \leq l \leq j$. Therefore, $H$ is the zero set of all $f_{j}$ for $f \in I(V)$ and $j \geq 1$. In particular, $H$ is an affine subscheme of Spec $\mathbf{k}\left[\underline{X}_{1}, \ldots, \underline{X}_{j}, \ldots\right]$.

For any integer $s \geq 0$, the canonical projection

$$
e_{s}: \mathbf{k}[[t]] \longrightarrow \mathbf{k}[[t]] /(t)^{s+1}
$$

induces a map $j^{s}: H \longrightarrow H_{s}$ where $H_{s}$ denotes the set of $\mathbf{k}$-local homomorphisms $\mathcal{O}_{V, O} \longrightarrow \mathbf{k}[[t]] /(t)^{s+1}$. Similarly, $H_{s}$ is the zero set of all $f_{j}$ for $f \in$ $I(V)$ and $1 \leq j \leq s$, hence an affine subscheme of $\mathbb{A}_{\mathbf{k}}^{s d}=\operatorname{Spec}\left[\underline{X}_{1}, \ldots, \underline{X}_{s}\right]$. The image $j^{s}(h)$ of $h \in H$ is called the $s$-jet (or $s$-truncation in [N]) of $h$. 
As a consequence of a theorem of Greenberg (a particular case of Artin's approximation theorem), $j^{s}(H)$ is a constructible subset (i.e. a finite union of locally closed subvarieties) of $H_{s}$, for every $s \geq 0$. Such a space has an image in the Grothendieck ring, $K_{0}\left(\mathcal{V}_{\mathbf{k}}\right)$, of k-varieties (i.e. reduced separated schemes of finite type over $\mathbf{k})$. Recall that, by definition, $K_{0}\left(\mathcal{V}_{\mathbf{k}}\right)$ is the ring generated by symbols $[X]$, for $X$ a $\mathbf{k}$-variety, modulo the relations $[X]=\left[X^{\prime}\right]$ if $X$ is $\mathbf{k}$-isomorphic to $X^{\prime},[X]=[X \backslash Y]+[Y]$ for $Y$ closed in $X$, and $[X] \times\left[X^{\prime}\right]=\left[X \times_{\mathbf{k}} X^{\prime}\right]$. As usual, we denote the class of the affine line $\mathbb{A}_{\mathbf{k}}^{1}$ by $\mathbb{L}$. Following Looijenga $[\mathrm{Lo}]$, we set $\mathcal{M}_{\mathbf{k}}:=K_{0}\left(\mathcal{V}_{\mathbf{k}}\right)\left[\mathbb{L}^{-1}\right]$.

Theorem (Denef-Loeser [D-L1]) Suppose that the ground field $\mathbf{k}$ is of characteristic zero. Then the power series

$$
P_{\text {geom }}(T):=\sum_{s \geq 0}\left[j^{s}(H)\right] T^{s}
$$

considered as a power series in $\mathcal{M}_{\mathbf{k}}[[T]]$ is rational. More precisely, there exist a polynomial $N(T) \in \mathcal{M}_{\mathbf{k}}[T]$ and finitely many integers $a_{i} \in \mathbb{Z}, b_{i}, d_{i} \in$ $\mathbb{Z}_{\geq 1}$, such that

$$
P_{\text {geom }}(T)=\frac{N(T)}{\prod_{i}\left(1-\mathbb{L}^{a_{i}} T^{b_{i}}\right)^{d_{i}}} .
$$

\section{Arcs on a toric surface singularity and their jets}

(3.1) Consider now an affine normal toric surface $V$ over an algebraically closed field $\mathbf{k}$. Let $T \cong\left(\mathbf{k}^{*}\right)^{2}$ be the 2-dimensional torus seating inside $V$. The ring of regular functions $\mathbf{k}[T]$ on $T$ is isomorphic to the ring of Laurent polynomials in 2 variables $\mathbf{k}\left[X_{1}, X_{1}^{-1}, X_{2}, X_{2}^{-1}\right]$. Recall that a one-parameter subgroup on $T$ is an homomorphism $\lambda$ from the multiplicative group $\mathbf{k}^{*}$ to $T$, and that there exists $n=\left(n_{1}, n_{2}\right) \in \mathbb{Z}^{2}$ such that $\lambda=\lambda_{n}$ where $\lambda_{n}$ is defined by

$$
X^{m} \circ \lambda_{n}(t)=t^{\langle m, n\rangle}
$$

for any $m=\left(m_{1}, m_{2}\right) \in \mathbb{Z}^{2}$, with $\langle m, n\rangle=\sum_{i=1,2} m_{i} n_{i}$.

In other words, the group of one-parameter subgroups on $T$ is a lattice $N \cong \mathbb{Z}^{2}$ dual to the lattice $M \cong \mathbb{Z}^{2}$ of exponents of monomials on $T$. Here is a list of basic facts of the theory of torus embeddings which we will use further. (See $[\mathrm{TE}]$ or $[\mathrm{O}]$ ).

i) There exists a strongly convex rational polyhedral cone $\sigma$ in $N_{\mathbb{R}}:=$ $N \otimes_{\mathbb{Z}} \mathbb{R} \cong \mathbb{R}^{2}$ such that $\lim _{t \rightarrow 0} \lambda_{n}(t)$ exists in $V$ if and only if $n \in \sigma \cap N$. 
ii) The ring of regular functions $\mathbf{k}[V]$ on $V$ is the $\mathbf{k}$-algebra $\mathbf{k}\left[\sigma^{\vee} \cap M\right]$ of the semigroup $\sigma^{\vee} \cap M$ where $\sigma^{\vee}=\left\{m \in M_{\mathbb{R}}:=M \otimes_{\mathbb{Z}} \mathbb{R}|m|_{\sigma} \geq 0\right\}$.

iii) Let $n, n^{\prime} \in \sigma \cap N$; then $\lambda_{n}$ and $\lambda_{n^{\prime}}$ tend to the same limit for $t \rightarrow 0$, if and only if $n$ and $n^{\prime}$ lie in the relative interior $\stackrel{\circ}{\tau}$ of a face $\tau$ of $\sigma$ (i. e. its topological interior in the $\mathbb{R}$-vector space $E_{\tau}$ spanned by $\tau$ ). As a consequence, the map $\tau \mapsto O_{\tau}:=T \cdot \lambda_{n}(0)$, for any $n \in \stackrel{\circ}{\tau} \cap N$, is a 1-1 correspondence from the set of faces of $\sigma$ to the set of $T$-orbits on $V$.

The orbit $O_{\tau}$ is isomorphic to a torus of dimension $2-\mathrm{rk}_{\mathbb{R}} E_{\tau}$. Since our surface $V$ is normal, its singular locus (if nonempty) consists of 0 -dimensional closed orbits. Therefore, $V$ contains such an orbit if and only if $\operatorname{dim} \sigma:=$ $\operatorname{dim} E_{\sigma}=2$ and, if this condition holds, then $O_{\sigma}$ is the only 0-dimensional orbit on $V$.

In the sequel, we will assume that $\operatorname{dim} \sigma=2$.

(3.2) An $\operatorname{arc} h$ on $V$ going through $O:=O_{\sigma}$ is a k-homomorphism of rings

$$
\mathbf{k}[V]=\mathbf{k}\left[\sigma^{\vee} \cap M\right] \longrightarrow \mathbf{k}[[t]]
$$

sending the maximal ideal $\mathcal{M}=\bigoplus_{m \in \sigma^{\vee} \cap M \backslash 0} \mathbf{k} X^{m}$ defining $O$ into $(t) \mathbf{k}[[t]]$.

Suppose that the image of $h$ meets the torus. Then the map

$$
\sigma^{\vee} \cap M \longrightarrow \mathbb{Z}_{\geq 0} \quad m \mapsto \operatorname{ord}_{t} X^{m} \circ h(t)
$$

is well defined. Indeed, for any such $m$, the zero set of $X^{m}$ is contained in $V \backslash T$. Since $T$ is dense in $V$, the $\mathbb{Z}$-module generated by $\sigma^{\vee} \cap M$ is $M$. So the above map extends to a linear form $\nu_{h}: M \longrightarrow \mathbb{Z}$, that is $\nu_{h} \in N$. We have $\left.\nu_{h}\right|_{\sigma^{\vee}} \geq 0$, hence $\nu_{h} \in \sigma^{\vee \vee}=\sigma$. Moreover, for any $m \neq 0$ in $\sigma^{\vee} \cap M$, we have $\nu_{h}(m)>0$, hence $\nu_{h} \in \stackrel{\circ}{\sigma}$.

Now, for any $m \in \sigma^{\vee} \cap M$, set $u_{h}(m)=X^{m} \circ h(t) t^{-\nu_{h}(m)}$. It follows from the definition of $\nu_{h}$ that $u_{h}(m)$ is a unit in $\mathbf{k}[[t]]$. As above, the homomorphism from $\sigma^{\vee} \cap M$ to the multiplicative group $U$ of units in $\mathbf{k}[[t]]$ extends to a group homomorphism from $M$ to $U$, and $u_{h}$ is nothing but an arc on $T$.

Conversely, we can recover an arc $h$ on $V$ going through $O$ whose image meets the torus from any pair $(\nu, u)$ with $\nu \in \stackrel{\circ}{\sigma} \cap N$ and $u$ in the set of arcs on $T$, by setting, for any $m \in \sigma^{\vee} \cap M$,

$$
X^{m} \circ h(t)=t^{\langle m, \nu\rangle} u(m) .
$$

Indeed, since $\nu \in \stackrel{\circ}{\sigma}$, we have $\langle m, \nu\rangle \geq 0$ and $\langle m, \nu\rangle>0$ if $m \neq 0$. We also have $X^{m} \circ h(t) \not \equiv 0$, hence the image of $h$ is not contained in $V \backslash T$, whose defining ideal is generated by monomials. 
To sum up, we have the following identification:

Proposition 3.3 Let $V$ be an affine normal toric surface with a 0-dimensional closed orbit $O$, and let $H^{*}$ denote the set of arcs on $V$ going through $O$ whose image meets the torus. Let $N$ be the lattice of one-parameter subgroups on $T, \sigma$ the 2-dimensional strongly convex rational polyhedral cone in $N_{\mathbb{R}}=$ $N \otimes_{\mathbb{Z}} \mathbb{R}^{2}$ enjoying property i) in (3.1), and $\stackrel{\circ}{\sigma}$ its relative interior. Let $H_{T}$ denote the set of arcs on $T$.

Then, the map $\left(\sigma^{0} \cap N\right) \times H_{T} \longrightarrow H^{*}$ sending $(\nu, u)$ to $h$ given by

$$
X^{m} \circ h(t)=t^{\langle m, \nu\rangle} u(m)
$$

for any monomial $X^{m}$ in $\mathbf{k}[V]$ is a one-to-one correspondence.

In the sequel, we will denote the pair coding $h \in H^{*}$ via (3.3.1) by $\left(\nu_{h}, u_{h}\right)$ and we will call $\nu_{h}$ the order form of $h$.

We can rephrase proposition 3.3 by saying that the family

$H_{\nu}^{*}:=\left\{h \in H^{*} \mid \nu_{h}=\nu\right\}=\left\{h \in H^{*} \mid \operatorname{ord}_{t} X^{m} \circ h(t)=\langle m, \nu\rangle, \forall m \in \sigma^{\vee} \cap M\right\}$,

for $\nu$ running over $\stackrel{\circ}{\sigma} \cap N$, is a partition of $H^{*}$ by subsets isomorphic to $H_{T}$. Note that $H_{T}=T \times \operatorname{Spec} \mathbf{k}\left[\left\{X_{1, s}, X_{2, s}\right\}_{s \geq 1}\right]$ with $X_{1, s}, X_{2, s}$ indeterminates.

(3.4) Our aim is to compute the image of $j^{s}(H)$ in $\mathcal{M}_{\mathbf{k}}$, for any integer $s \geq 0$. To do so, we have to exhibit a finite partition of $j^{s}(H)$ by constructible subsets whose image in $\mathcal{M}_{\mathbf{k}}$ is easily computed. The first elementary observation is that $j^{s}(H)=j^{s}\left(H^{*}\right)$ (see lemma 3.5 below). We will extract a finite partition of $j^{s}\left(H^{*}\right)$ from the infinite family $\left\{j^{s}\left(H_{\nu}^{*}\right)\right\}_{\nu \in \sigma \cap \cap}$. We immediately see that $j^{s}\left(H_{\nu}^{*}\right)=\{0\}$ if $\min \left\{\langle m, \nu\rangle \mid m \in \sigma^{\vee} \cap M \backslash 0\right\}>s$. We will prove that otherwise, $j^{s}\left(H_{\nu}^{*}\right)$ is isomorphic to the product of a 1 or 2-dimensional torus by an affine space (see lemmas 3.6, 3.8 and 3.9 below).

Lemma 3.5 For any integer $s \geq 0$, we have $j^{s}(H)=j^{s}\left(H^{*}\right)$.

Proof: We may assume that $\sigma=\mathbb{R}_{\geq 0} \epsilon_{1}+\mathbb{R}_{\geq 0}\left(p \epsilon_{1}+q \epsilon_{2}\right)$ where $\left(\epsilon_{1}, \epsilon_{2}\right)$ is the canonical basis of $N \cong \mathbb{Z}^{2}$ and $p, q$ are relatively prime integers with $0 \leq p<q$. All we have to prove is that, given $h \in H$ whose image lies in $V \backslash T$, we can find $h^{\prime} \in H^{*}$ with the same $s$-jet. Here $V \backslash T$ is the union of $O:=O_{\sigma}$ and of two 1-dimensional orbits corresponding to the 1-dimensional faces of $\sigma$. We may assume that $h$ factors through $Y:=\overline{O_{\mathbb{R}_{>0} \epsilon_{1}}}$. The ideal defining $Y$ in $V$ is $\oplus_{m \in\left(\sigma^{\vee} \backslash \tau^{\perp}\right) \cap M} \mathbf{k} X^{m}$ with $\tau:=\mathbb{R}_{\geq 0} \epsilon_{1}$. Hence, for any $m \in\left(\sigma^{\vee} \backslash \tau^{\perp}\right) \cap M$, we have $X^{m} \circ h(t)=0$. 
Let $\left(e_{1}, e_{2}\right)$ be the dual basis of $\left(\epsilon_{1}, \epsilon_{2}\right)$. We have $\sigma^{\vee}=\mathbb{R}_{\geq 0}\left(q e_{1}-p e_{2}\right)+$ $\mathbb{R}_{\geq 0} e_{2}$ and $\tau^{\perp}=\mathbb{R} e_{2}$. By proposition 3.3, we have to find $\nu \in \stackrel{\circ}{\sigma} \cap N$ and $u \in H_{T}$ such that

$$
\langle m, \nu\rangle>s, \quad \forall m \in\left(\sigma^{\vee} \backslash \tau^{\perp}\right) \cap M
$$

and

$$
t^{\left\langle e_{2}, \nu\right\rangle} u\left(e_{2}\right)-X^{e_{2}} \circ h(t) \in(t)^{s+1} .
$$

Set $\nu_{2}:=\operatorname{ord}_{t} X^{e_{2}} \circ h(t)$. If $s<\nu_{2}\left(\right.$ or $\left.\nu_{2}=+\infty\right)$, then $j^{s}(h)=0$, and we have $j^{s}\left(h^{\prime}\right)=0$ for any $h^{\prime} \in H_{\nu}^{*}$ with $\min _{m \in \sigma^{\vee} \cap M \backslash 0}\langle m, \nu\rangle>s$. If $1 \leq \nu_{2} \leq s$, choose an integer $\nu_{1}>s+\frac{p}{q} \nu_{2}$ and set $\nu:=\nu_{1} e_{1}+\nu_{2} e_{2}$. For any $m=m_{1} e_{1}+m_{2} e_{2} \in\left(\sigma^{\vee} \backslash \tau^{\perp}\right) \cap M$, we have $p m_{1}+q m_{2} \geq 0$ and $m_{1} \geq 1$, hence $\langle m, \nu\rangle=m_{1} \nu_{1}+m_{2} \nu_{2} \geq \frac{m_{1}}{q}\left(q \nu_{1}-p \nu_{2}\right)>s$. Besides, since $\nu_{2} \geq 1$ and $q \nu_{1}-p \nu_{2}>s \geq 0$, we have $\nu \in \stackrel{\circ}{\sigma}$. Now, $X^{e_{2}} \circ h(t) t^{-\nu_{2}}$ is a unit $u_{2}$ in $\mathbf{k}[[t]]$. Let $u \in H_{T}$ mapping $m \in M$ to $u_{2}^{\left\langle m, \epsilon_{2}\right\rangle}$. Then we have $t^{\left\langle e_{2}, \nu\right\rangle} u\left(e_{2}\right)=t^{\nu_{2}} u_{2}=X^{e_{2}} \circ h(t)$, therefore the pair $(\nu, u)$ satisfies our requirements.

We analyze arc and jet spaces through computations involving coordinates on $V$. Here, the $T$-action on $V$ provides us with a natural system of coordinates on $V$ vanishing at $O$, namely $\left(X^{\mu}\right)_{\mu \in G}^{\vee}$ where $\stackrel{\vee}{G}$ is the minimal system of generators of the semigroup $\sigma^{\vee} \cap M \backslash 0$. Recall that $\stackrel{\vee}{G}$ consists of the integral points on the compact edges of the boundary polygon, $\partial \mathcal{P}$, of the convex hull $\mathcal{P}$ of $\sigma^{\vee} \cap M \backslash 0$, and that, given $\mu_{1}, \mu_{2}$ in $\stackrel{\vee}{G},\left(\mu_{1}, \mu_{2}\right)$ is a $\mathbb{Z}$-basis of $M$ if and only if the segment $\left[\mu_{1}, \mu_{2}\right]$ lies in $\partial \mathcal{P}$ and contains no points in $M$ other than the vertices (see [GS1] or [O]).

The following lemma is preliminary.

Lemma 3.6 Fix $\nu \in \stackrel{\circ}{\sigma} \cap N$ and let $H_{\nu}^{*}:=\left\{h \in H^{*} \mid \nu_{h}=\nu\right\}$. Suppose that there exists $\mu_{1}, \mu_{2}$ in $\stackrel{\vee}{G}$ such that $\left(\mu_{1}, \mu_{2}\right)$ is a $\mathbb{Z}$-basis of $M$ and that, for any $\mu \in \stackrel{\vee}{G}$ distinct from $\mu_{1}, \mu_{2}$, the inequality

$$
\langle\mu, \nu\rangle \geq \sup _{i=1,2}\left\langle\mu_{i}, \nu\right\rangle
$$

holds. Then $j^{s}\left(H_{\nu}^{*}\right)$ is isomorphic to

$$
\begin{array}{ll}
\{0\} & \text { if } s<\inf _{i=1,2}\left\langle\mu_{i}, \nu\right\rangle ; \\
\mathbf{k}^{*} \times \mathbb{A}_{\mathbf{k}}^{s-\inf _{i=1,2}\left\langle\mu_{i}, \nu\right\rangle} & \text { if }\left\langle\mu_{1}, \nu\right\rangle \neq\left\langle\mu_{2}, \nu\right\rangle \text { and } \\
& \quad \inf _{i=1,2}\left\langle\mu_{i}, \nu\right\rangle \leq s<\sup _{i=1,2}\left\langle\mu_{i}, \nu\right\rangle ; \\
\mathbf{k}^{*^{2}} \times \mathbb{A}_{\mathbf{k}}^{2 s-\left\langle\mu_{1}+\mu_{2}, \nu\right\rangle} & \text { if } \sup _{i=1,2}\left\langle\mu_{i}, \nu\right\rangle \leq s .
\end{array}
$$


Proof: By proposition 3.3, the map $H_{T} \longrightarrow H_{\nu}^{*}$ sending $u$ to $h$ given by

$$
X^{\mu} \circ h(t)=t^{\langle\mu, \nu\rangle} u(\mu) \quad \forall \mu \in \stackrel{\vee}{G}
$$

is an isomorphism. Now, any $\mu \in \stackrel{\vee}{G}$ is a linear combination $\mu=a_{1} \mu_{1}+a_{2} \mu_{2}$ with $a_{i} \in \mathbb{Z}$. We have $u(\mu)=\prod_{i=1,2} u\left(\mu_{i}\right)^{a_{i}}$.

Set $u(\mu)=\sum_{r>0} u(\mu ; r) t^{r}, u\left(\mu_{i}\right)=\sum_{r>0} u\left(\mu_{i} ; r\right) t^{r}, i=1,2$. Since $u(\mu), u\left(\mu_{i}\right), i=1,2$, are units in $\mathbf{k}[[t]]$, for any integer $r \geq 0, u(\mu ; r)$ is a polynomial function of $u\left(\mu_{i} ; 0\right)^{-1}, u\left(\mu_{i} ; 0\right), u\left(\mu_{i} ; 1\right), \cdots, u\left(\mu_{i} ; r\right), i=1,2$.

We have

(1) $X^{\mu} \circ h(t)=\sum_{r \geq 0} u(\mu ; r) t^{\langle\mu, \nu\rangle+r}$;

(2) $X^{\mu_{i}} \circ h(t)=\sum_{r \geq 0} u\left(\mu_{i} ; r\right) t^{\left\langle\mu_{i}, \nu\right\rangle+r} \quad i=1,2$.

For simplicity, assume that $\left\langle\mu_{1}, \nu\right\rangle \leq\left\langle\mu_{2}, \nu\right\rangle$. By assumption, for any $\mu \neq \mu_{1}, \mu_{2}$ in $\stackrel{\vee}{G}$, we have $\left\langle\mu_{2}, \nu\right\rangle \leq\langle\mu, \nu\rangle$. Therefore, if $0 \leq s<\left\langle\mu_{1}, \nu\right\rangle$, we have $j^{s}(h)=0$, while, if $\left\langle\mu_{1}, \nu\right\rangle \leq s<\left\langle\mu_{2}, \nu\right\rangle$, then $X^{\mu_{1}} \circ h(t)$ is the only component of $h$ having a nonzero $s$-jet. In view of (2), this proves the first two isomorphisms stated in the lemma.

Now, suppose that $\left\langle\mu_{2}, \nu\right\rangle \leq s$. By (1), for $0 \leq r \leq s$, the coefficient of $t^{r}$ in $X^{\mu} \circ h(t)$ is 0 if $r<\langle\mu, \nu\rangle$ and $u(\mu ; r-\langle\mu, \nu\rangle)$ if $\langle\mu, \nu\rangle \leq r$. In both cases, it is a polynomial function of $u\left(\mu_{i} ; 0\right)^{-1}, u\left(\mu_{i} ; 0\right), \ldots, u\left(\mu_{i} ; r-\langle\mu, \nu\rangle\right), i=1,2$. Since $\left\langle\mu_{i}, \nu\right\rangle \leq\langle\mu, \nu\rangle, i=1,2$, these functions give the last isomorphism in the lemma, in view of $(2)$.

The next step will be to prove that $\mathbb{Z}$-basis of $M$ enjoying the inequality (3.6.1) do exist for any $\nu \in \sigma^{\circ} \cap N$, and to control how they depend on $\nu$. To begin with, given $\nu \in \stackrel{\circ}{\sigma} \cap N$, we are looking for the two smallest integers occuring in the family $\stackrel{\vee}{G}(\nu):=\{\langle\mu, \nu\rangle\}_{\mu \in \underset{G}{\vee}}$.

Note that two integers in $\stackrel{\vee}{G}(\nu)$ may coincide. We will show that the resulting numerical functions on $\stackrel{\circ}{\sigma} \cap N$ are piecewise linear functions, i.e. linear functions on the cones of a fan $\widehat{\Sigma}$ which is a subdivision of $\sigma$, and that, for each $\widehat{\sigma} \in \widehat{\Sigma}$, the pair of linear functions on $N$ thus obtained is a $\mathbb{Z}$-basis of $M$ which satisfies the hypothesis of lemma 3.6 with respect to any $\nu \in \widehat{\sigma} \cap N$. We will show that, if the characteristic of $\mathbf{k}$ is zero, the corresponding equivariant modification $\pi: \widehat{V} \longrightarrow V$ is the minimal one which dominates the blowing-up with center $O$ and the Nash blowing-up ${ }^{1}$ of $V$.

\footnotetext{
${ }^{1}$ See appendix.
} 
To prove this, we first need to recall some elementary facts about blowingups of monomial ideals.

(3.7) Consider an ideal $\mathcal{A}$ in $\mathbf{k}[V]=\mathbf{k}\left[\sigma^{\vee} \cap M\right]$ generated by monomials $\left(X^{m}\right)_{m \in A}$. It is easily verified that, for any $n \in \sigma$, we have

$$
\operatorname{ord} \mathcal{A}(n):=\inf _{m \in A}\langle m, n\rangle=\inf _{m \in \mathcal{N}_{\mathcal{A}}}\langle m, n\rangle=: h_{\mathcal{N}_{\mathcal{A}}}(n)
$$

where $\mathcal{N}_{\mathcal{A}}$ is the convex hull of $\cup_{m \in A}\left(m+\sigma^{\vee}\right)$ and $h_{\mathcal{N}_{\mathcal{A}}}$ denotes its support function defined by the right hand side equality.

For any nonempty face $F$ of $\mathcal{N}_{\mathcal{A}}, \sigma_{F}:=\left\{n \in \sigma \mid\langle m, n\rangle=h_{\mathcal{N}_{\mathcal{A}}}(n)\right.$ for any $m \in F\}$ is a strongly convex cone in $\sigma$, and the resulting family $\left\{\sigma_{F}\right\}$ is a fan $\Sigma_{\mathcal{A}}$ which subdivides $\sigma$. The corresponding equivariant modification $\pi_{\mathcal{A}}: V_{\mathcal{A}} \longrightarrow V$ is the blowing-up with center $\mathcal{A}$ followed by normalization. (See [TE, chap. I, sec. 2]). In the sequel, we will call $\mathcal{N}_{\mathcal{A}}\left(\operatorname{resp} . \Sigma_{\mathcal{A}}\right)$ the Newton polygon (resp. Newton fan) of $\mathcal{A}$.

Note that, for any $m \in F \cap M$, the ideal $\mathcal{A} \mathbf{k}\left[V_{\sigma_{F}}\right]$ is generated by $X^{m}$, and that $h_{\mathcal{N}_{\mathcal{A}}}$ coincides with the linear function $m$ on $\sigma_{F}$. Here $V_{\sigma_{F}}$ denotes the affine chart of $V_{\mathcal{A}}$ whose ring of functions $\mathbf{k}\left[V_{\sigma_{F}}\right]$ is $\mathbf{k}\left[\sigma_{F}^{\vee} \cap M\right]$. For any $n \in \sigma_{F}$, we thus have:

$$
\operatorname{ord} \mathcal{A}(n):=\inf _{m \in A}\langle m, n\rangle=\langle m, n\rangle \quad \forall m \in F \cap M .
$$

The above equality applied to the maximal ideal $\mathcal{M}=\left(X^{\mu}\right)_{\mu \in G} v$ defining $O$ expresses that $\min _{\mu \in G}\langle\mu, \nu\rangle$ is a piecewise linear function. More precisely, we get:

Lemma 3.8 In the above notation, the following holds:

i) The Newton polygon of $\mathcal{M}$ is the convex hull $\mathcal{P}$ of $\sigma^{\vee} \cap M \backslash 0$.

ii) Let $\Sigma^{\prime}$ be the Newton fan of $\mathcal{M}$ and let $\sigma_{S} \in \Sigma^{\prime}$ as defined in (3.7) from a vertex $S$ of $\mathcal{P}$. For any $\nu \in \sigma_{S}$, we have

$$
\operatorname{ord} \mathcal{M}(\nu):=\inf _{\mu \in G}\langle\mu, \nu\rangle=\langle S, \nu\rangle \text {. }
$$

iii) Let $\pi^{\prime}: V^{\prime} \longrightarrow V$ be the equivariant modification corresponding to the fan $\Sigma^{\prime}$ subdivision of $\sigma$. Then $\pi^{\prime}$ is the blowing-up with center $O$.

Note that there is no need to normalize after blowing-up here. Singularities of toric surfaces are rational surface singularities and this fact follows from [L, proposition 8.1]. 
G. González-Sprinberg ([GS1]) has proved that, if $\mathbf{k}$ is a field of characteristic 0, the Nash blowing-up of $V$ is the blowing-up with respect to the ideal $\mathcal{J}_{l}$ in $\mathbf{k}[V]=\mathbf{k}\left[\sigma^{\vee} \cap M\right]$ generated by $X^{\mu+\mu^{\prime}}$, where $\mu, \mu^{\prime}$ is running over the set of pairs of $\mathbb{R}$-linearly independent elements in $\stackrel{\vee}{G}$. (See appendix for an alternative proof). In this paper, we call this ideal the logarithmicjacobian ideal. Note that we have ord $\mathcal{J}_{l} \geq 2$ ord $\mathcal{M}$. By applying (3.7.1) to $\mathcal{J}_{l} \mathcal{M}^{-1} \mathbf{k}\left[V_{\sigma_{S}}\right]$, we will get that the restriction of $\min _{\mu \in G \backslash S}\langle\mu, \nu\rangle$ to $\sigma_{S}$ is a piecewise linear function. This goes as follows:

Lemma 3.9 Order the points $\mu_{0}, \ldots, \mu_{e+1}$ in the minimal generating system $\stackrel{\vee}{G}$ in such a way that, for any $i, 0 \leq i \leq e$, the segment $\left[\mu_{i}, \mu_{i+1}\right]$ lies in $\partial \mathcal{P}$ and contains no points in $M$ other that its vertices. For a vertex $S$ labelled $\mu_{i}$, set $S^{-}:=\mu_{i-1}$ if $i \neq 0$ and $S^{+}:=\mu_{i+1}$ if $i \neq e+1$. Then:

i) The Newton polygon of $\mathcal{J}_{l} \mathcal{M}^{-1} \mathbf{k}\left[V_{\sigma_{S}}\right]$ is the convex hull $\mathcal{P}_{S}$ of $\left[S^{-} S^{+}\right]+$ $\sigma_{S}^{\vee}\left(\right.$ resp. $\left.S^{+}+\sigma_{S}^{\vee}, S^{-}+\sigma_{S}^{\vee}\right)$ if $S \neq \mu_{0}, \mu_{e+1}\left(\right.$ resp. $\left.S=\mu_{0}, S=\mu_{e+1}\right)$.

ii) Let $\Sigma_{S}$ be the Newton fan of $\mathcal{J}_{l} \mathcal{M}^{-1} \mathbf{k}\left[V_{\sigma_{S}}\right]$, and let $\sigma_{S^{*}} \in \Sigma_{S}$ with $*=+$ or - as defined in (3.7). For any $\nu \in \sigma_{S^{*}}$, we have

$$
\operatorname{ord} \mathcal{J}_{l}(\nu)-\operatorname{ord} \mathcal{M}(\nu)=\inf _{\substack{v \\ \mu \backslash S}}\langle\mu, \nu\rangle=\left\langle S^{*}, \nu\right\rangle .
$$

iii) Let $\widehat{\Sigma}$ be the fan subdivision of $\Sigma^{\prime}$ obtained by removing $\sigma_{S}$ from $\Sigma^{\prime}$ and adjoining the cones in $\Sigma_{S}$ for every vertex $S$ of $\mathcal{P}$. Let $\pi: \widehat{V} \longrightarrow V$ be the composition of the blowing-up $\pi^{\prime}: V^{\prime} \longrightarrow V$ with center $O$ with the equivariant modification $\widehat{V} \longrightarrow V^{\prime}$ corresponding to the subdivision $\widehat{\Sigma}$ of $\Sigma^{\prime}$. Then $\pi$ is the minimal modification with $\widehat{V}$ normal factoring through the blowing-up with center $O$ and the Nash blowing-up of $V$.

Proof: We have $\mathcal{M} \mathbf{k}\left[V_{\sigma_{S}}\right]=\left(X^{S}\right)$. Therefore $\mathcal{J}_{l} \mathcal{M}^{-1} \mathbf{k}\left[V_{\sigma_{S}}\right]$ is generated by $\left(X^{\mu_{j}+\mu_{l}-S}\right)_{0 \leq j, l \leq e+1, j \neq l}$. Hence the ideal $\mathcal{I}_{S}$ generated by $\left(X^{\mu}\right)_{\mu \in G \backslash S}$ in $\mathbf{k}\left[V_{\sigma_{S}}\right]$ is contained in $\mathcal{J}_{l} \mathcal{M}^{-1} \mathbf{k}\left[V_{\sigma_{S}}\right]$, the Newton polygon $\tilde{\mathcal{N}}$ of $\mathcal{I}_{S}$ is contained in the Newton polygon $\mathcal{N}$ of $\mathcal{J}_{l} \mathcal{M}^{-1} \mathbf{k}\left[V_{\sigma_{S}}\right]$ and, for any $\nu \in \sigma_{S}$, we have by lemma 3.8,

$$
\inf _{\substack{v \\ \mu \in G \backslash S}}\langle\mu, \nu\rangle \geq \inf _{j \neq l}\left\langle\mu_{j}+\mu_{l}-S, \nu\right\rangle=\text { ord } \mathcal{J}_{l}(\nu)-\text { ord } \mathcal{M}(\nu) .
$$

On the other hand, for $j \neq l$, either $\mu_{j}$ or $\mu_{l}$ is distinct from $S=\mu_{i}$. Assume $j \neq i$, then, by lemma 3.8 again, we get that

$$
\left\langle\mu_{j}+\mu_{l}, \nu\right\rangle \geq\left\langle\mu_{j}, \nu\right\rangle+\langle S, \nu\rangle \geq \inf _{\mu \in G \backslash S}\langle\mu, \nu\rangle+\langle S, \nu\rangle .
$$


So, for $\nu \in \sigma_{S}$, we have

$$
\text { ord } \mathcal{J}_{l}(\nu)-\text { ord } \mathcal{M}(\nu)=\inf _{\mu \in G \backslash S}\langle\mu, \nu\rangle \text {. }
$$

By (3.7), equality (3.9.1) means that the support functions of the Newton polygons $\mathcal{N}$ and $\tilde{\mathcal{N}}$ coincide. Therefore, the supporting lines in the direction $n$ to $\mathcal{N}$ and $\widetilde{\mathcal{N}}$ respectively

$$
L_{n, \mathcal{N}(\operatorname{resp} . \tilde{\mathcal{N}})}=\left\{m \in M_{\mathbb{R}} \mid\langle m, n\rangle=h_{\mathcal{N}(\operatorname{resp} . \tilde{\mathcal{N}})}(n)\right\}
$$

coincide. Let $L_{n}$ denote this line. To get $\mathcal{N}=\widetilde{\mathcal{N}}$, it is enough to prove that $\mathcal{N} \subseteq \widetilde{\mathcal{N}}$. Consider a vertex $Q$ of $\mathcal{N}$ and choose $n$ in the relative interior of $\sigma_{Q} \in \Sigma_{S}$. We have $\mathcal{N} \cap L_{n}=\{Q\}$. But, since $\widetilde{\mathcal{N}} \subseteq \mathcal{N}$, the nonempty face $\widetilde{\mathcal{N}} \cap L_{n}$ of $\widetilde{\mathcal{N}}$ is contained in $\{Q\}$, hence it is $\{Q\}$. Therefore $Q$ is a vertex of $\widetilde{\mathcal{N}}$ and we get $\mathcal{N} \subseteq \tilde{\mathcal{N}}$.

It remains to prove that

$$
\widetilde{\mathcal{N}}:=\operatorname{Conv} \bigcup_{\mu \in G \backslash S}\left(\mu+\sigma_{S}^{\vee}\right)=\mathcal{P}_{S} .
$$

Assume first that $S \neq \mu_{0}$ and $\mu_{e+1}$. The inclusion $\widetilde{\mathcal{N}} \supseteq \mathcal{P}_{S}$ is clear because $S^{-}, S^{+} \in G$ and $\mathcal{P}_{S}=\operatorname{Conv}\left[\cup_{*=+,-}\left(S^{*}+\sigma_{S}^{\vee}\right)\right]$. To get the opposite inclusion, it is enough to prove that any $\mu \in \stackrel{\vee}{G} \backslash S$ lies in $\mathcal{P}_{S}$. Any such $\mu$ is a point of $\partial \mathcal{P}$, hence of $\mathcal{P}$. But we have $\mathcal{P} \subseteq S+\sigma_{S}^{\vee}$ (in fact, $\mathcal{P}$ is the intersection of the family of $R+\sigma_{R}^{\vee}$ for $R$ running over the set of vertices of $\mathcal{P}$ ). Since $S+\sigma_{S}^{\vee}$ is the union of $\left[S^{-} S^{+}\right]+\sigma_{S}^{\vee}$ and the triangle with vertices $S, S^{-}, S^{+}$, and the only points of $\stackrel{\vee}{G}$ in this triangle are its vertices, we get the conclusion.

Assume now that $S=\mu_{0}$. The inclusion $\tilde{N} \supseteq \mathcal{P}_{S}$ is again clear. As above, we have $\mathcal{P} \subseteq S+\sigma_{S}^{\vee}$. Here $S+\sigma_{S}^{\vee}$ is the union of $S^{+}+\sigma_{S}^{\vee}$ and of the Minkowsky sum $\theta$ of the segment $\left[S, S^{+}\right]=\left[\mu_{0}, \mu_{1}\right]$ and of $\mathbb{R}_{\geq 0} \mu_{0}$. The only points of $\stackrel{\vee}{G}$ in $\theta$ are $S$ and $S^{+}$, hence $\stackrel{\vee}{G} \backslash S$ is a subset of $S^{+}+\sigma_{S}^{\vee}=\mathcal{P}_{S}$. This completes the proof of $i$ ).

Now, in view of i), assertion ii) is an immediate consequence of (3.9.1), (3.9.2) and (3.7.1) applied to the ideal $\mathcal{I}_{S}:=\left(X^{\mu}\right)_{\mu \in G \backslash S}^{\vee}$ in $\mathbf{k}\left[V_{\sigma_{S}}\right]$.

As for iii), we know by lemma 3.8, that $\pi^{\prime}: V^{\prime} \longrightarrow V$ is the blowingup with center $\mathcal{M}$. Now, it follows from (3.7) applied to $\mathcal{J}_{l} \mathcal{M}^{-1} \mathbf{k}\left[V_{\sigma_{S}}\right]$ 
for every vertex $S$ of $\mathcal{P}$, that the modification $\widehat{V} \longrightarrow V^{\prime}$ is the blowingup of $V^{\prime}$ with center $\mathcal{J}_{l} \mathcal{M}^{-1} \mathcal{O}_{V}$, followed by normalization. Hence, the composition $\pi: \widehat{V} \longrightarrow V$ is the blowing-up of $V$ with center $\mathcal{J}_{l} \mathcal{M}$ followed by normalization, i.e. the minimal modification with $\widehat{V}$ normal factoring through the blowing-up with center $O$ and the Nash blowing-up of $V$ in characteristic 0 .

Since, for any vertex $S$ of $\mathcal{P}$, the pair $\left(S, S^{*}\right)$ with $S^{*}=S^{-}$or $S^{+}$is a $\mathbb{Z}$-basis of $M$, and $\sigma$ is covered by the family of $\sigma_{S^{*}}$, lemma 3.6 combined with lemmas 3.8 and 3.9 computes the isomorphism class of $j^{s}\left(H_{\nu}^{*}\right)$ for every $\nu \in \stackrel{\circ}{\sigma} \cap N$. The last step is to exhibit the partition of $j^{s}(H)$ by finitely many $j^{s}\left(H_{\nu}^{*}\right)$ that we have announced in (3.4).

This requires to introduce some additional notation.

Lemma-Definition 3.10 For any integer $s \geq 0$, let

$$
\begin{aligned}
& \sigma_{0}(s):=\{n \in \stackrel{\circ}{\sigma} \mid \text { ord } \mathcal{M}(n)>s\} \\
& \sigma_{1}(s):=\left\{n \in \stackrel{\circ}{\sigma} \mid \text { ord } \mathcal{M}(n) \leq s<\text { ord } \mathcal{J}_{l}(n)-\text { ord } \mathcal{M}(n)\right\} \\
& \sigma_{2}(s):=\left\{n \in \stackrel{\circ}{\sigma} \mid \text { ord } \mathcal{J}_{l}(n)-\text { ord } \mathcal{M}(n) \leq s\right\}
\end{aligned}
$$

Then we have,

i) The family $\left\{\sigma_{i}(s)\right\}_{0 \leq i \leq 2}$ is a partition of $\stackrel{\circ}{\sigma}$.

ii) $\sigma_{2}(s) \cap N$ is a finite set (empty if $\left.s=0\right)$.

iii) For $s \geq 1, \sigma_{1}(s)$ is the disjoint union of $\sigma_{1}(s) \cap \stackrel{\circ}{\sigma}_{S}$ for $S$ running over the set of vertices of $\mathcal{P}$.

Proof: By the definition of the logarithmic-jacobian ideal $\mathcal{J}_{l}$, we have ord $\mathcal{J}_{l} \geq 2$ ord $\mathcal{M}$, hence i). The set $\sigma_{2}(s)$ is relatively compact, hence ii). The 1-dimensional cones in $\Sigma^{\prime}$ intersecting $\stackrel{\circ}{\sigma}$ are the cones $\sigma_{F}$ for $F$ running over the set of 1-dimensional compact edges of $\mathcal{P}$. Pick any such $F$ and choose a pair $\left(S, S^{+}\right)$on it. By $(3.7 .1)$, for any $n \in \sigma_{F}$, we have $(S, n)=$ $\left(S^{+}, n\right)$. Now $\sigma_{F}$ is a face of both $\sigma_{S}$ and $\sigma_{S^{+}}$. By lemmas 3.8 and 3.9, this equality is equivalent to saying that ord $\mathcal{M}(n)=$ ord $\mathcal{J}_{l}(n)-$ ord $\mathcal{M}(n)$ and implies that $\sigma_{F} \cap \sigma_{1}(s)=\emptyset$, hence iii).

Note that, for $s \geq 1$ and $S \neq \mu_{0}, \mu_{e+1}, \sigma_{1}(s) \cap \stackrel{\circ}{\sigma}_{S}$ is relatively compact, hence $\sigma_{1}(s) \cap \stackrel{\circ}{\sigma}_{S} \cap N$ is a finite set. This does not hold for $S=\mu_{0}$ or $\mu_{e+1}$. 
Now, we may state the main result of this section.

Proposition 3.11 Let $H$ (resp. $H^{*}$ ) denote the set of arcs on $V$ going through $O$ (resp. whose image meets the torus).

For each $h \in H^{*}$, let $\nu_{h}$ denote its order form (see (3.3)). For each integer $s \geq 0$, set $H_{i}^{*}(s)=\left\{h \in H^{*} \mid \nu_{h} \in \sigma_{i}(s)\right\}, 0 \leq i \leq 2$, and for each $\nu \in \stackrel{\circ}{\sigma} \cap N$, set $H_{\nu}^{*}=\left\{h \in H^{*} \mid \nu_{h}=\nu\right\}$. Then,

i) For each $s \geq 0$, the family $\left\{j^{s}\left(H_{i}^{*}(s)\right)\right\}_{0 \leq i \leq 2}$ is a partition of $j^{s}(H)$.

ii) a) We have $j^{s}\left(H_{0}^{*}(s)\right)=\{0\}$.

b) The family $\left\{j^{s}\left(H_{\nu}^{*}\right)\right\}_{\nu \in \sigma_{1}(s)}$ is a covering of $j^{s}\left(H_{1}^{*}(s)\right)$ by constructible sets. Given $\nu, \nu^{\prime} \in \sigma_{1}(s)$, we have $j^{s}\left(H_{\nu}^{*}\right) \cap j^{s}\left(H_{\nu^{\prime}}^{*}\right) \neq \emptyset$ if and only if there exists a vertex $S$ of $\mathcal{P}$ such that $\nu, \nu^{\prime} \in \stackrel{\circ}{\sigma}_{S}$ and ord $\mathcal{M}(\nu)=$ ord $\mathcal{M}\left(\nu^{\prime}\right)$. And if this holds, then we have

$$
j^{s}\left(H_{\nu}^{*}\right)=j^{s}\left(H_{\nu^{\prime}}^{*}\right) \cong \mathbf{k}^{*} \times \mathbb{A}_{\mathbf{k}}^{s-\operatorname{ord} \mathcal{M}(\nu)} .
$$

c) The family $\left\{j^{s}\left(H_{\nu}^{*}\right)\right\}_{\nu \in \sigma_{2}(s)}$ is a partition of $j^{s}\left(H_{2}^{*}(s)\right)$ by constructible sets and, for any $\nu \in \sigma_{2}(s)$, we have

$$
j^{s}\left(H_{\nu}^{*}\right) \cong \mathbf{k}^{*^{2}} \times \mathbb{A}_{\mathbf{k}}^{2 s-\text { ord } \mathcal{J}_{l}(\nu)} .
$$

Proof: By lemma 3.5, we have $j^{s}(H)=j^{s}\left(H^{*}\right)$ for any $s \geq 0$. Now $\left\{\sigma_{i}(s)\right\}_{0 \leq i \leq 2}$ is a covering of $\stackrel{\circ}{\sigma}$, therefore the 1-1 correspondence between $H^{*}$ and $(\stackrel{\circ}{\sigma} \cap N) \times H_{T}$ shown in proposition 3.3, implies that $\left\{j^{s}\left(H_{i}^{*}(s)\right)\right\}_{0 \leq i \leq 2}$ is a covering of $j^{s}\left(H^{*}\right)$.

For every $h \in H^{*}$, let

$$
\stackrel{\vee}{G}(h ; s):=\left\{\mu \in \stackrel{\vee}{G} \mid j^{s}\left(X^{\mu} \circ h(t)\right) \neq 0\right\}=\left\{\mu \stackrel{\vee}{G} \mid\left\langle\mu, \nu_{h}\right\rangle \leq s\right\} .
$$

Note that, if the $s$-jets of $h, h^{\prime} \in H^{*}$ coincide, then $\stackrel{\vee}{G}(h ; s) \stackrel{\vee}{G}\left(h^{\prime} ; s\right)$. Now, since the maximal ideal $\mathcal{M}$ defining $O$ is generated by $\left(X^{\mu}\right)_{\mu \in G}$ and by definition ord $\mathcal{M}(\nu):=\inf _{\mu \in G}\langle\mu, \nu\rangle$, for any $h \in H^{*}$, we have the five equivalences below:

$$
\stackrel{\vee}{G}(h ; s)=\emptyset \Leftrightarrow j^{s}(h)=0 \Leftrightarrow \operatorname{ord} \mathcal{M}\left(\nu_{h}\right)>s \Leftrightarrow \nu_{h} \in \sigma_{0}(s) \Leftrightarrow h \in H_{0}^{*}(s) .
$$

This proves a) in ii). 
Now suppose that $\stackrel{\vee}{G}(h ; s)$ contains a single element, say $\mu_{i}$, with $0 \leq$ $i \leq e+1$ (see lemma 3.9). We have

$$
\text { ord } \mathcal{M}\left(\nu_{h}\right):=\inf _{\mu \in G}\left\langle\mu, \nu_{h}\right\rangle=\inf _{\mu \in G(h ; s)}\left\langle\mu, \nu_{h}\right\rangle \leq s
$$

By (3.7) and lemma $3.8 \mathrm{i}$ ), ord $\mathcal{M}$ is nothing but the support function $h_{\mathcal{P}}$ of the convex hull $\mathcal{P}$ of $\sigma^{\vee} \cap M \backslash 0$. Hence our assumption implies that the face of $\mathcal{P}$ contained in the supporting line

$$
L_{\nu_{h}}:=\left\{m \in M_{\mathbb{R}} \mid\left\langle m, \nu_{h}\right\rangle=h_{\mathcal{P}}\left(\nu_{h}\right)\right\}
$$

in the direction $\nu_{h}$ is the singleton $\left\{\mu_{i}\right\}$. So $\mu_{i}$ is a vertex $S$ of $\mathcal{P}$ and $\nu_{h} \in \stackrel{\circ}{\sigma}_{S}$. Moreover, by lemma 3.9 ii), we conclude that

$$
\text { ord } \mathcal{J}_{l}\left(\nu_{h}\right)-\text { ord } \mathcal{M}\left(\nu_{h}\right)=\inf _{\substack{v \\ \mu \in S}}\left\langle\mu, \nu_{h}\right\rangle>s .
$$

Therefore, $\nu_{h} \in \sigma_{1}(s)$ and $h \in H_{1}^{*}(s)$.

Now, consider $h^{\prime} \in H^{*}$ such that $j^{s}(h)=j^{s}\left(h^{\prime}\right)$. It follows from the equality $\stackrel{\vee}{G}(h ; s)=\stackrel{\vee}{G}\left(h^{\prime} ; s\right)=\{S\}$ and the above argument that $\nu_{h^{\prime}} \in \stackrel{\circ}{\sigma}_{S} \cap \sigma_{1}(s)$ and that

$$
j^{s}\left(X^{S} \circ h(t)\right)=j^{s}\left(X^{S} \circ h^{\prime}(t)\right) \neq 0
$$

thus

$$
\operatorname{ord}_{t} X^{S} \circ h(t)=\left\langle S, \nu_{h}\right\rangle=\operatorname{ord}_{t} X^{S} \circ h^{\prime}(t)=\left\langle S, \nu_{h^{\prime}}\right\rangle
$$

or equivalently, by lemma 3.8 , ord $\mathcal{M}\left(\nu_{h}\right)=$ ord $\mathcal{M}\left(\nu_{h^{\prime}}\right)$.

Next suppose that $\stackrel{\vee}{G}(h ; s)$ contains at least two elements. Since $\nu_{h} \in \stackrel{\circ}{\sigma}$, the face $F$ of $\mathcal{P}$ contained in the supporting line $L_{\nu_{h}}$ is either a vertex or a 1-dimensional compact edge of $\mathcal{P}$. For any $m \in F$, we have

$$
h_{\mathcal{P}}\left(\nu_{h}\right)=\left\langle m, \nu_{h}\right\rangle=\inf _{\mu \in G}\left\langle\mu, \nu_{h}\right\rangle=\inf _{\substack{v \\ \mu \in G(h ; s)}}\left\langle\mu, \nu_{h}\right\rangle \leq s .
$$

Hence $F \cap M \subseteq \stackrel{\vee}{G}(h ; s)$ and $\nu_{h} \in \sigma_{F}$. Choose a vertex $S$ of $F$; in particular $S \in \stackrel{\vee}{G}(h ; s)$. Now our assumption implies that inf ${ }_{\mu \in G \backslash S}\left\langle\mu, \nu_{h}\right\rangle \leq s$. Since $\sigma_{F} \subseteq \sigma_{S}$, by lemma $3.9 \mathrm{ii)}$ again, we have

$$
\text { ord } \mathcal{J}_{l}\left(\nu_{h}\right)-\operatorname{ord} \mathcal{M}\left(\nu_{h}\right)=\inf _{\mu \in G \backslash S}\left\langle\mu, \nu_{h}\right\rangle=\left\langle S^{*}, \nu_{h}\right\rangle \leq s
$$

for a suitable value - or + of $*$. Hence $\nu_{h} \in \sigma_{2}(s)$, i.e. $h \in H_{2}^{*}(s)$ and $S, S^{*} \in \stackrel{\vee}{G}(h ; s)$. 
As above, for $h^{\prime} \in H^{*}$ such that $j^{s}(h)=j^{s}\left(h^{\prime}\right)$, we deduce from the equality $\stackrel{\vee}{G}(h ; s)=\stackrel{\vee}{G}\left(h^{\prime} ; s\right)$ that $\nu_{h^{\prime}} \in \sigma_{2}(s)$. Moreover $\stackrel{\vee}{G}(h ; s)$ contains a $\mathbb{Z}$-basis $\left(S, S^{*}\right)$ of $M$. For $\mu=S$ or $S^{*}$, we have

$$
j^{s}\left(X^{\mu} \circ h(t)\right)=j^{s}\left(X^{\mu} \circ h^{\prime}(t)\right) \neq 0
$$

thus

$$
\operatorname{ord}_{t} X^{\mu} \circ h(t)=\left\langle\mu, \nu_{h}\right\rangle=\operatorname{ord}_{t} X^{\mu} \circ h^{\prime}(t)=\left\langle\mu, \nu_{h^{\prime}}\right\rangle
$$

from which we conclude that $\nu_{h}=\nu_{h^{\prime}}$. Here the basis enjoying the hypothesis of lemma 3.6 with respect to $\nu_{h}=\nu_{h^{\prime}}=\nu$ is $\left(S, S^{*}\right)$. We have

$$
\left\langle S+S^{*}, \nu\right\rangle=\operatorname{ord} \mathcal{M}(\nu)+\left(\operatorname{ord} \mathcal{J}_{l}(\nu)-\text { ord } \mathcal{M}(\nu)\right)=\text { ord } \mathcal{J}_{l}(\nu) \text {. }
$$

In addition, since $\left\{\sigma_{i}(s)\right\}_{0 \leq i \leq 2}$ is a partition of $\stackrel{\circ}{\sigma}$, the above discussion shows that, for $i=0,1$ (resp. 2), we have $\nu_{h} \in \sigma_{i}(s)$ if and only if the set $\stackrel{\vee}{G}(h ; s)$ has $i$ (resp. at least $i$ ) elements. This completes the proof of $\mathrm{i}$ ), and b), c) in ii), except for the constructibility property of $j^{s}\left(H_{\nu}^{*}\right)$.

By definition (see lemma 3.9) $\left(X^{\mu_{i}}\right)_{0 \leq i \leq e+1}$ is a system of coordinates on $V$ which vanish at $O$. Recall that the set $H_{s}$ of k-local homomorphisms $\mathcal{O}_{V, O} \longrightarrow \mathbf{k}[[t]] /(t)^{s+1}$ is an affine subscheme of $\mathbb{A}_{\mathbf{k}}^{s(e+2)}$ and that $j^{s}(H)=$ $j^{s}\left(H^{*}\right)$ is a constructible subset of $H_{s}$. Consider $\nu \in \sigma_{i}(s) \cap N$, with $i=1$ or 2. For any $h \in H^{*}$, we have $j^{s}(h) \in j^{s}\left(H_{\nu}\right)$ if and only if, for $i=$ $0, \ldots, e+1$,

$$
\operatorname{ord}_{t} X^{\mu_{i}} \circ h(t)=\left\{\begin{array}{cc}
\left\langle\mu_{i}, \nu\right\rangle & \text { if }\left\langle\mu_{i}, \nu\right\rangle \leq s \\
>s & \text { otherwise }
\end{array}\right.
$$

These conditions define a constructible subset in $\mathbb{A}_{\mathbf{k}}^{s(e+2)}$. Hence $j^{s}\left(H_{\nu}^{*}\right)$ is constructible.

Corollary 3.12 Let $\mathcal{M}$ (resp. $\mathcal{J}_{l}$ ) be the maximal ideal defining $O$ in $V$ (resp. the logarithmic-jacobian ideal) and let ord $\mathcal{M}$ (resp. ord $\mathcal{J}_{l}$ ): $\sigma \longrightarrow$ $\mathbb{Z}_{\geq 0}$ denote the corresponding order functions as defined in (3.7).

For any integer $s \geq 0$, let $\sigma_{i}(s), i=1,2$, be the subset of $\stackrel{\circ}{\sigma}$ defined in lemma-definition 3.10. Denote by $\left[\sigma_{1}(s) \cap N\right]$ (resp. $[\nu]$ ) the image of $\sigma_{1}(s) \cap N$ (resp. $\nu \in \sigma_{1}(s) \cap N$ ) in the quotient of $\sigma_{1}(s)$ by the equivalence relation: $n \sim n^{\prime}$ if there exists a vertex $S$ of $\mathcal{P}$ such that $n, n^{\prime} \in \sigma_{S}$ and ord $\mathcal{M}(n)=$ ord $\mathcal{M}\left(n^{\prime}\right)$. Then we have

$$
\left[j^{s}(H)\right]=1+(\mathbb{L}-1) \sum_{[\nu] \in\left[\sigma_{1}(s) \cap N\right]} \mathbb{L}^{s-\text { ord } \mathcal{M}(\nu)}+(\mathbb{L}-1)^{2} \sum_{\nu \in \sigma_{2}(s) \cap N} \mathbb{L}^{2 s-\text { ord } \mathcal{J}_{l}(\nu)}
$$

in the Grothendieck group of $\mathbf{k}$-varieties $K_{0}\left(\mathcal{V}_{\mathbf{k}}\right)$. 


\section{The series}

(4.1) We keep the notation of section 3. In this section, we will give an explicit formula for

$$
P_{\text {geom }, \mathrm{V}}(T):=\sum_{s \geq 0}\left[j^{s}(H)\right] T^{s}
$$

in terms of the cone $\sigma$ attached to $V$ (see (3.1)). It follows from corollary 3.12 that

$$
P_{\text {geom }, \mathrm{V}}(T):=P_{0}(T)+P_{1}(T)+P_{2}(T)
$$

where

$$
\begin{aligned}
P_{0}(T) & :=\sum_{s \geq 0} T^{s}=\frac{1}{1-T} \\
P_{1}(T) & :=(\mathbb{L}-1) \sum_{s \geq 0} T^{s} \sum_{[\nu] \in\left[\sigma_{1}(s) \cap N\right]} \mathbb{L}^{s-\text { ord } \mathcal{M}(\nu)} \\
P_{2}(T) & :=(\mathbb{L}-1)^{2} \sum_{s \geq 0} T^{s} \sum_{\nu \in \sigma_{2}(s) \cap N} \mathbb{L}^{2 s-\text { ord } \mathcal{J}_{l}(\nu)} .
\end{aligned}
$$

We first proceed to compute $P_{2}(T)$. Recall that

$$
\sigma_{2}(s)=\left\{\nu \in \stackrel{\circ}{\sigma} \mid \text { ord } \mathcal{J}_{l}(\nu)-\text { ord } \mathcal{M}(\nu) \leq s\right\}
$$

Therefore

$$
\begin{aligned}
P_{2}(T) & =(\mathbb{L}-1)^{2} \sum_{\nu \in \dot{\sigma} \cap N} \sum_{s \geq \operatorname{ord}} \sum_{\mathcal{J}_{l}(\nu)-\text { ord } \mathcal{M}(\nu)} \mathbb{L}^{2 s-\text { ord } \mathcal{J}_{l}(\nu)} T^{s} \\
& =(\mathbb{L}-1)^{2} \sum_{\nu \in \dot{\sigma} \cap N} \mathbb{L}^{\operatorname{ord} \mathcal{J}_{l}(\nu)-2 \operatorname{ord} \mathcal{M}(\nu)} T^{\operatorname{ord} \mathcal{J}_{l}(\nu)-\operatorname{ord} \mathcal{M}(\nu)}\left(\sum_{s \geq 0} \mathbb{L}^{2 s} T^{s}\right) \\
& =\frac{(\mathbb{L}-1)^{2}}{1-\mathbb{L}^{2} T} N_{2}(T)
\end{aligned}
$$

with

$$
N_{2}(T):=\sum_{\nu \in \sigma \cap N} \mathbb{L}^{\text {ord } \mathcal{J}_{l}(\nu)-2 \operatorname{ord} \mathcal{M}(\nu)} T^{\text {ord } \mathcal{J}_{l}(\nu)-\operatorname{ord} \mathcal{M}(\nu)}
$$

Let $\widehat{\Sigma}$ be the fan subdivision of $\Sigma$ introduced in lemma 3.9. By the inclusionexclusion principle, we have

$$
N_{2}(T)=\sum_{\widehat{\sigma} \in \widehat{\Sigma}}(-1)^{\operatorname{dim} \widehat{\sigma}} N_{2, \widehat{\sigma}}(T)
$$

with

$$
N_{2, \widehat{\sigma}}(T):=\sum_{\nu \in \widehat{\sigma} \cap \circ \cap \cap N} \mathbb{L}^{\text {ord } \mathcal{J}_{l}(\nu)-2 \operatorname{ord} \mathcal{M}(\nu)} T^{\text {ord } \mathcal{J}_{l}(\nu)-\operatorname{ord} \mathcal{M}(\nu)}
$$


We know by lemmas 3.8 and 3.9 that the functions ord $\mathcal{M}$ and ord $\mathcal{J}_{l}-$ ord $\mathcal{M}$ coincide with linear forms on each $\widehat{\sigma} \in \widehat{\Sigma}$. More precisely, for $\widehat{\sigma}=\sigma_{S^{*}}$ as in lemma 3.9 , we have

ord $\mathcal{M}(\nu)=\langle S, \nu\rangle \quad$ and $\quad$ ord $\mathcal{J}_{l}(\nu)-$ ord $\mathcal{M}(\nu)=\left\langle S^{*}, \nu\right\rangle \quad \forall \nu \in \sigma_{S^{*}}$.

Thus, for any face $\tau$ of $\sigma_{S^{*}}$, we have

$$
N_{2, \tau}(T)=\sum_{\nu \in \tau \cap \circ \circ \cap N} \mathbb{L}^{\left\langle S^{*}-S, \nu\right\rangle} T^{\left\langle S^{*}, \nu\right\rangle} .
$$

Since $\left(S, S^{*}\right)$ is a $\mathbb{Z}$-basis of $M=\operatorname{Hom}_{\mathbb{Z}}(N, \mathbb{Z})$, the map $\varphi: N \longrightarrow \mathbb{Z} \times \mathbb{Z}$ sending $\nu$ to $\left(\left\langle S^{*}-S, \nu\right\rangle,\left\langle S^{*}, \nu\right\rangle\right)$ is an isomorphism.

For a vertex $S$ of $\mathcal{P}$ in the relative interior of $\sigma^{\vee}$ (i. e. $S \neq \mu_{0}, \mu_{e+1}$ ), we have by definition (see again lemma 3.9)

$$
\sigma_{S^{*}}=\left\{n \in N_{\mathbb{R}} \mid\left\langle S^{*}-S, n\right\rangle \geq 0 \text { and } *\left\langle S^{-}-S^{+}, n\right\rangle \geq 0\right\} .
$$

There exists an integer $c_{S} \geq 1$ such that $S^{+}+S^{-}=\left(c_{S}+2\right) S$. So the cone $\sigma_{S^{*}}$ is mapped by $\varphi$ to the cone

$$
\begin{aligned}
\rho_{S} & =\left\{\left(\alpha_{1}, \alpha_{2}\right) \in \mathbb{R} \times \mathbb{R} / \alpha_{1} \geq 0, c_{S} \alpha_{2}-\left(c_{S}+2\right) \alpha_{1} \geq 0\right\} \\
& =\mathbb{R}_{\geq 0}\left(c_{S}, c_{S}+2\right)+\mathbb{R}_{\geq 0}(0,1)
\end{aligned}
$$

and

$$
N_{2, \sigma_{S^{*}}}(T)=\sum_{\left(\alpha_{1}, \alpha_{2}\right) \in \rho_{S} \cap \mathbb{Z}^{2}} \mathbb{L}^{\alpha_{1}} T^{\alpha_{2}}-1 .
$$

The primitive vector on $\mathbb{R}_{\geq 0}\left(c_{S}, c_{S}+2\right)$ is $\left(q_{S}, q_{S}+p_{S}\right)$ with $q_{S}=c_{S}, p_{S}=2$ if $c_{S}$ is odd, $q_{S}=\frac{c_{S}}{2}, p_{S}=1$ if $c_{S}$ is even. It is easily verified that

$$
N_{2, \sigma_{S^{*}}}(T)=\frac{P_{S}(\mathbb{L}, T)}{(1-T)\left(1-\mathbb{L}^{q_{S}} T^{q_{S}+p_{S}}\right)}-1
$$

where

$$
P_{S}(\mathbb{L}, T):=\sum_{\Pi_{S} \cap \mathbb{Z}^{2}} \mathbb{L}^{\alpha_{1}} T^{\alpha_{2}}
$$

and

$$
\Pi_{S}:=\left\{\lambda_{1}\left(q_{S}, q_{S}+p_{S}\right)+\lambda_{2}(0,1) \mid 0 \leq \lambda_{i}<1, i=1,2\right\}
$$

i. e.

$$
P_{S}(\mathbb{L}, T)=\sum_{0 \leq \alpha<q_{S}} \mathbb{L}^{\alpha} T^{\left\lceil\frac{\alpha\left(q_{S}+p_{S}\right)}{q_{S}}\right\rceil}
$$

where $\left\lceil\frac{\alpha\left(q_{S}+p_{S}\right)}{q_{S}}\right\rceil$ denotes the least integer greater than or equal to $\frac{\alpha\left(q_{S}+p_{S}\right)}{q_{S}}$. 
We also get that

$$
N_{2, \tau}(T)=\frac{\mathbb{L}^{q_{S}} T^{q_{S}+p_{S}}}{\left(1-\mathbb{L}^{q_{S}} T^{q_{S}+p_{S}}\right)} \quad\left(\operatorname{resp} \cdot \frac{T}{(1-T)}\right)
$$

if $\tau$ is the 1-dimensional face of $\sigma_{S^{*}}$ in $\widehat{\Sigma} \backslash \Sigma^{\prime}$ (resp. in $\Sigma^{\prime}$ ).

For $S=\mu_{0}, \mu_{e+1}$, we have:

$$
\sigma_{S^{*}}=\left\{n \in N_{\mathbb{R}} \mid\left\langle S^{*}-S, n\right\rangle \geq 0 \text { and }\langle S, n\rangle \geq 0\right\} .
$$

Here the cone $\sigma_{S^{*}}$ is mapped by $\varphi$ onto the cone

$$
\begin{aligned}
\rho & =\left\{\left(\alpha_{1}, \alpha_{2}\right) \in \mathbb{R} \times \mathbb{R} \mid \alpha_{1} \geq 0, \alpha_{2}-\alpha_{1} \geq 0\right\} \\
& =\mathbb{R}_{\geq 0}(1,1)+\mathbb{R}_{\geq 0}(0,1)
\end{aligned}
$$

and

$$
\begin{aligned}
N_{2, \sigma_{S^{*}}}(T) & =\sum_{\left(\alpha_{1}, \alpha_{2}\right) \in \rho \cap \mathbb{Z}^{2}} \mathbb{L}^{\alpha_{1}} T^{\alpha_{2}}-\sum_{\left(\alpha_{1}, \alpha_{2}\right) \in \mathbb{R}_{\geq 0}(1,1) \cap \mathbb{Z}^{2}} \mathbb{L}^{\alpha_{1}} T^{\alpha_{2}} \\
& =\frac{1}{(1-T)(1-\mathbb{L} T)}-\frac{1}{(1-\mathbb{L} T)}=\frac{T}{(1-T)(1-\mathbb{L} T)} .
\end{aligned}
$$

Summing up, this gives

$$
\begin{aligned}
N_{2}(T)= & \frac{T(1+\mathbb{L} T)}{(1-T)(1-\mathbb{L} T)} \\
& +\sum_{S \in \mathcal{S}}\left(\frac{2 P_{S}(\mathbb{L}, T)}{(1-T)\left(1-\mathbb{L}^{\left.q_{S} T^{q_{S}+p_{S}}\right)}\right.}-\frac{1}{1-T}-\frac{1}{1-\mathbb{L}^{q_{S}} T^{q_{S}+p_{S}}}\right)
\end{aligned}
$$

where $\mathcal{S}$ is the set of vertices of the convex hull $\mathcal{P}$ of $\sigma^{\vee} \cap M \backslash 0$ in the relative interior of $\sigma^{\vee}$.

We now compute $P_{1}(T)$. Recall that $\sigma_{1}(0)=\emptyset$ and that, for $s \geq 1$, $\sigma_{1}(s)$ is the disjoint union of $\sigma_{1}(s) \cap \stackrel{\circ}{\sigma}_{S}$ for $S$ running over the set of vertices of $\mathcal{P}$. Moreover, the quotient space $\left[\sigma_{1}(s) \cap N\right]$ is the disjoint union of $\left[\sigma_{1}(s) \cap \stackrel{\circ}{\sigma}_{S} \cap N\right]$ for $S \in \mathcal{S}$, and, for $n, n^{\prime} \in \sigma_{1}(s) \cap \stackrel{\circ}{\sigma}_{S}$, we have $[n]=\left[n^{\prime}\right]$ if and only if ord $\mathcal{M}(n)=\langle S, n\rangle=$ ord $\mathcal{M}\left(n^{\prime}\right)=\left\langle S, n^{\prime}\right\rangle$.

Lemma 4.2 Let $(r, s)$ be a pair of nonnegative integers. For $S \in \mathcal{S}$ (resp. $\left.S \in \partial \sigma^{\vee}\right)$, the following conditions are equivalent:

i) There exists $\nu \in \sigma_{1}(s) \cap \stackrel{\circ}{\sigma}_{S} \cap N$ such that $\langle S, \nu\rangle=s-r$.

ii) $(r+1, s+1) \in \rho_{S}=\mathbb{R}_{\geq 0}\left(q_{s}, q_{S}+p_{S}\right)+\mathbb{R}_{\geq 0}(0,1) \quad$ (resp. $\quad(r, s) \in$ $\left.\rho \backslash \mathbb{R}_{\geq 0}(1,1)=\mathbb{R}_{>0}(1,1)+\mathbb{R}_{\geq 0}(0,1)\right)$. 
Proof: Assume that $S \in \mathcal{S}$. It follows from the definition of $\widehat{\Sigma}$ (see lemma 3.9), from lemma-definition 3.10 and from the explicit description of $\sigma_{S^{*}}$ by inequalities given above that

$$
\begin{aligned}
\sigma_{1}(s) & \cap \stackrel{\circ}{\sigma}_{S}=\bigcup_{* \in\{+,-\}}\left(\sigma_{1}(s) \cap \sigma_{S}^{*}\right) \\
& =\bigcup_{*=+,-}\left\{n \in N_{\mathbb{R}} \mid\langle S, n\rangle \leq s<\left\langle S^{*}, n\right\rangle, *\left\langle S^{-}-S^{+}, n\right\rangle \geq 0\right\} \\
& =\bigcup_{*=+,-}\left\{n \in N_{\mathbb{R}} \mid\langle S, n\rangle \leq s<\left\langle S^{*}, n\right\rangle,\left(c_{S}+2\right)\langle S, n\rangle \geq 2\left\langle S^{*}, n\right\rangle\right\} .
\end{aligned}
$$

Since $\left(S, S^{*}\right)$ is a $\mathbb{Z}$-basis of $M=\operatorname{Hom}_{\mathbb{Z}}(N, \mathbb{Z})$, condition i) holds if and only if there exists $q \in \mathbb{Z}$ such that

$$
s<q \text { and }\left(c_{S}+2\right)(s-r)-2 q \geq 0
$$

or equivalently

$$
2(s+1) \leq\left(c_{S}+2\right)(s-r)
$$

i.e.

$$
c_{S}(s+1)-\left(c_{S}+2\right)(r+1) \geq 0
$$

or

$$
q_{S}(s+1)-\left(q_{S}+p_{S}\right)(r+1) \geq 0 .
$$

Since $r \geq 0$, the above inequality is equivalent to condition ii).

For $S$ on $\partial \sigma^{\vee}$, we have $\sigma_{S}=\sigma_{S}^{*}$ with $*=+\left(\right.$ resp. - ) if $S=\mu_{0}$ (resp. $\mu_{e+1}$ ) (see again lemma 3.9). Hence

$$
\sigma_{1}(s) \cap \stackrel{\circ}{\sigma}_{S}=\left\{n \in N_{\mathbb{R}} \mid\langle S, n\rangle \leq s<\left\langle S^{*}, n\right\rangle,\langle S, n\rangle>0\right\} .
$$

For $(r, s) \in \mathbb{Z}_{\geq 0} \times \mathbb{Z}_{\geq 0}$, condition i) holds if and only if $s>r$, i.e. $(r, s) \in$ $\rho \backslash \mathbb{R}_{\geq 0}(1,1)$.

It follows immediately from lemma 4.2 that

$$
P_{1}(T)=(\mathbb{L}-1)\left(2 P_{1, \rho}+\sum_{S \in \mathcal{S}} P_{1, \rho_{S}}\right)
$$

with

$$
\begin{aligned}
P_{1, \rho} & :=\sum_{(r, s) \in \rho \cap \mathbb{Z}^{2}} \mathbb{L}^{r} T^{s}-\sum_{(r, s) \in \mathbb{Z}_{\geq 0}(1,1)} \mathbb{L}^{r} T^{s} \\
& =\frac{1}{(1-T)(1-\mathbb{L} T)}-\frac{1}{1-\mathbb{L} T}=\frac{T}{1-\mathbb{L} T}
\end{aligned}
$$


600 M. Lejeune-Jalabert and A. J. Reguera

and

$$
\begin{aligned}
P_{1, \rho_{S}} & :=\mathbb{L}^{-1} T^{-1}\left(\sum_{(r, s) \in \rho_{S} \cap \mathbb{Z}^{2}} \mathbb{L}^{r} T^{s}-\sum_{s \in \mathbb{Z}_{\geq 0}} T^{s}\right) \\
& =\frac{\mathbb{L}^{-1} T^{-1}}{1-T}\left(\frac{P_{S}(\mathbb{L}, T)}{1-\mathbb{L}^{q_{S}} T^{q_{S}+p_{S}}}-1\right) .
\end{aligned}
$$

To eliminate artificial poles in $\mathbb{L}, T$ we use the identity:

$$
\begin{aligned}
& \frac{P_{S}(\mathbb{L}, T)}{(1-T)\left(1-\mathbb{L}^{q_{S}} T^{q_{S}+p_{S}}\right)}+\frac{Q_{S}(\mathbb{L}, T)}{(1-\mathbb{L})\left(1-\mathbb{L}^{q_{S}} T^{q_{S}+p_{S}}\right)}= \\
& =\frac{1}{(1-T)(1-\mathbb{L})}+\frac{1}{\left(1-\mathbb{L}^{q_{S}} T^{q_{S}+p_{S}}\right)}
\end{aligned}
$$

where

$$
Q_{S}(\mathbb{L}, T):=\sum_{(r, s) \in \Xi_{S} \cap \mathbb{Z}^{2}} \mathbb{L}^{r} T^{s}
$$

and

$$
\Xi_{S}:=\left\{\lambda_{1}\left(q_{S}, q_{S}+p_{S}\right)+\lambda_{2}(1,0) \mid 0 \leq \lambda_{i}<1, i=1,2\right\}
$$

i.e.

$$
Q_{S}(\mathbb{L}, T)=\sum_{0 \leq r<q_{S}+p_{S}} \mathbb{L}^{\left\lceil\frac{r q_{S}}{q_{S}+p_{S}}\right\rceil} T^{r}
$$

We get that

$$
(\mathbb{L}-1) P_{1, \rho_{S}}=\frac{\mathbb{L}^{-1} T^{-1}\left(Q_{S}-1+\mathbb{L}^{q_{S}+1} T^{q_{S}+p_{S}}\right)}{1-\mathbb{L}^{q_{S}} T^{q_{S}+p_{S}}}-\frac{1}{1-T} .
$$

Note that

$$
Q_{S}^{\prime}:=Q_{S}-1+\mathbb{L}^{q_{S}+1} T^{q_{S}+p_{S}}=\sum_{(r, s) \in \Xi_{S}^{\prime} \cap \mathbb{Z}^{2}} \mathbb{L}^{r} T^{s}
$$

with

$$
\Xi_{S}^{\prime}=\left\{\lambda_{1}\left(q_{S}, q_{S}+p_{S}\right)+\lambda_{2}(1,0) \mid 0<\lambda_{i} \leq 1, i=1,2\right\}
$$

and that

$$
R_{S}:=\mathbb{L}^{-1} T^{-1} Q_{S}^{\prime}=\sum_{0 \leq r<p_{S}+q_{S}} \mathbb{L}^{\left\lfloor\frac{(r+1) q_{S}}{q_{S}+p_{S}}\right\rfloor} T^{r}
$$

where $\left\lfloor\frac{(r+1) q_{S}}{q_{S}+p_{S}}\right\rfloor$ denotes the greatest integer less than or equal to $\frac{(r+1) q_{S}}{q_{S}+p_{S}}$. Finally, we get

$$
P_{1}(T)=\frac{2(\mathbb{L}-1) T}{(1-T)(1-\mathbb{L} T)}+\sum_{S \in \mathcal{S}}\left(\frac{R_{S}(\mathbb{L}, T)}{1-\mathbb{L}^{q_{S}} T^{q_{S}+p_{S}}}-\frac{1}{1-T}\right) .
$$


Now, it is easily checked from the above computations that

$$
\begin{aligned}
& P_{\text {geom }}(T)=P_{0}(T)+P_{1}(T)+P_{2}(T)=\frac{1}{1-\mathbb{L}^{2} T} \\
& +\frac{(\mathbb{L}-1)^{2}}{1-\mathbb{L}^{2} T} \sum_{S \in \mathcal{S}}\left(\frac{2 P_{S}(\mathbb{L}, T)}{(1-T)\left(1-\mathbb{L}^{q_{S}} T^{q_{s}+p_{S}}\right)}-\frac{1}{1-T}-\frac{1}{1-\mathbb{L}^{q_{S}} T^{q_{S}+p_{S}}}\right) \\
& +\sum_{S \in \mathcal{S}}\left(\frac{R_{S}(\mathbb{L}, T)}{1-\mathbb{L}^{q_{S}} T^{q_{S}+p_{S}}}-\frac{1}{1-T}\right) .
\end{aligned}
$$

(4.3) A natural question arises: What are the actual poles of $P_{\text {geom }}(T)$ ? Let us consider $1-T$ first. We have

$$
\begin{aligned}
& P_{\text {geom }}(T)=\frac{1}{1-\mathbb{L}^{2} T}-\frac{(\mathbb{L}-1)^{2}}{1-\mathbb{L}^{2} T} \sum_{S \in \mathcal{S}} \frac{1}{1-\mathbb{L}^{q_{S}} T^{q_{S}+p_{S}}}+\sum_{S \in \mathcal{S}} \frac{R_{S}}{1-\mathbb{L}^{q_{S}} T^{q_{s}+p_{S}}} \\
& +\frac{1}{(1-T)\left(1-\mathbb{L}^{2} T\right)} \sum_{S \in \mathcal{S}}\left(\frac{2(\mathbb{L}-1)\left[(\mathbb{L}-1) P_{S}+1-\mathbb{L}^{q_{S}} T^{q_{S}+p_{S}}\right]}{1-\mathbb{L}^{q_{S}} T^{q_{S}+p_{S}}}-\mathbb{L}^{2}(1-T)\right)
\end{aligned}
$$

But the identity (4.2.1) is equivalent to

$$
(\mathbb{L}-1) P_{S}+1-\mathbb{L}^{q_{S}} T^{q_{S}+p_{S}}=(1-T)\left(Q_{S}+\mathbb{L}-1\right) .
$$

Therefore $1-T$ is not a pole of $P_{\text {geom }}(T)$.

More precisely, we get

$$
\begin{aligned}
P_{\text {geom }}(T)= & \frac{1}{1-\mathbb{L}^{2} T}+\sum_{S \in \mathcal{S}} \frac{2(\mathbb{L}-1) Q_{S}+(\mathbb{L}-1)^{2}-\mathbb{L}^{2}\left(1-\mathbb{L}^{q_{S}} T^{q_{S}+p_{S}}\right)}{\left(1-\mathbb{L}^{2} T\right)\left(1-\mathbb{L}^{q_{S}} T^{q_{S}+p_{S}}\right)} \\
& +\sum_{S \in \mathcal{S}} \frac{R_{S}}{1-\mathbb{L}^{q_{S}} T^{q_{S}+p_{S}}} .
\end{aligned}
$$

Finally, using (4.2.2) and (4.2.3), we obtain

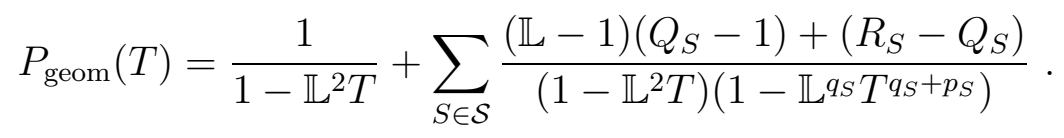

Note that

$$
Q_{S}=R_{S}=\sum_{0 \leq r \leq q_{S}} \mathbb{L}^{r} T^{r} \quad \text { if } p_{S}=1
$$

and

$$
\begin{aligned}
Q_{S} & =\sum_{0 \leq r \leq\left\lceil\frac{q_{S}}{2}\right\rceil} \mathbb{L}^{r} T^{r}+\sum_{\left\lceil\frac{q_{S}}{2}\right\rceil<r \leq q_{S}+1} \mathbb{L}^{r-1} T^{r} \\
R_{S} & =\sum_{0 \leq r<\left\lceil\frac{q_{S}}{2}\right\rceil} \mathbb{L}^{r} T^{r}+\sum_{\left\lceil\frac{q_{S}}{2}\right\rceil \leq r \leq q_{S}+1} \mathbb{L}^{r-1} T^{r} \quad \text { if } p_{S}=2,
\end{aligned}
$$


hence

$$
R_{S}-Q_{S}=-(\mathbb{L}-1) \mathbb{L}^{\left\lfloor\frac{q_{S}}{2}\right\rfloor} T^{\left\lceil\frac{q_{S}}{2}\right\rceil} \quad \text { if } p_{S}=2
$$

In both cases $\mathbb{L}-1$ factors $R_{S}-Q_{S}$.

It is immediately seen on the expression (4.3.1) that $1-\mathbb{L}^{q_{S}} T^{q_{S}+p_{S}}$ is a pole of $P_{\text {geom }}(T)$ for every $S \in \mathcal{S}$. Indeed, $\mathbb{Z}[\mathbb{L}, T]$ is a unique factorization domain and, since $q_{S}$ and $q_{S}+p_{S}$ are relatively prime, $1-\mathbb{L}^{q_{S}} T^{q_{S}+p_{S}}$ is irreducible. This polynomial of degree $q_{S}+p_{S}$ with respect to $T$ may not be a divisor of $(\mathbb{L}-1)\left(Q_{S}-1\right)+\left(R_{S}-Q_{S}\right)$, which has degree $q_{S}+p_{S}-1$ with respect to $T$.

As for $1-\mathbb{L}^{2} T$, it follows from [D-L1, theorem 7.1], that $P_{\text {geom }}(T)(1-$ $\mathbb{L}^{2} T$ ) evaluated at $T=\mathbb{L}^{-2}$ is the motivic volume $\mu(H)$ which is nonzero. So, $1-\mathbb{L}^{2} T$ is a simple pole. Here these facts are easily verified as follows: In view of [D-L1, (3.2.2)-(3.2.3)], we have

$$
\mu(H)=\mu\left(H^{*}\right)=\sum_{\nu \in \sigma \cap N} \mu\left(H_{\nu}^{*}\right)
$$

by proposition 3.3. Proposition 3.11 ii) c) expresses that $H_{\nu}^{*}$ is stable at level ord $\mathcal{J}_{l}(\nu)-$ ord $\mathcal{M}(\nu)$ and that

$$
\mu\left(H_{\nu}^{*}\right)=(\mathbb{L}-1)^{2} \mathbb{L}^{- \text {ord } \mathcal{J}_{l}(\nu)}
$$

(definitions are given in [D-L1, (2.4), (2.7) and (3.1)]). Note that here we adopt Loojenga's definition ([Lo, p. 04]), i. e. we multiply Denef-Loeser measure by $\mathbb{L}^{2}$. Now $1-\mathbb{L}^{2} T$ does not appear among the poles of $P_{1}(T)$ and $P_{0}(T)$. Hence, using the notation of (4.1) we have

$$
\begin{aligned}
\left.P_{\text {geom }}(T)\left(1-\mathbb{L}^{2} T\right)\right|_{T=\mathbb{L}^{-2}} & =(\mathbb{L}-1)^{2} N_{2}\left(\mathbb{L}^{-2}\right) \\
& =(\mathbb{L}-1)^{2} \sum_{\nu \in \sigma \circ N} \mathbb{L}^{- \text {ord } \mathcal{J}_{l}(\nu)}=\mu(H) .
\end{aligned}
$$

We conclude from (4.3.1), (4.3.2) and (4.3.3) that

$$
\mu(H)=1+(\mathbb{L}-1) \sum_{S \in \mathcal{S}} \frac{M_{S}(\mathbb{L})}{\mathbb{L}^{q_{S}+2 p_{S}}-1}
$$

with

$$
M_{S}(\mathbb{L})=\left\{\begin{array}{cc}
\mathbb{L}^{2}+\cdots+\mathbb{L}^{q_{S}+1} & \text { if } p_{S}=1 \\
\mathbb{L}^{2}+\cdots+\mathbb{L}^{q_{S}+3}-2 \mathbb{L}^{2+\frac{q_{S}+1}{2}} & \text { if } p_{S}=2
\end{array}\right.
$$


(4.4) The set $\mathcal{S}$ and the integers $p_{S}, p_{S}+q_{S}$, for $S \in \mathcal{S}$, introduced in (4.1), which occur in the formulas (4.3.1) and (4.3.5) for $P_{\text {geom }}(T)$ and $\mu(H)$ respectively have the following geometric significance: It follows from (3.7), lemma 3.8 and (3.1) iii), that the 0-dimensional orbits on the blowing-up $V^{\prime}$ of $V$ with center $O$ are the points $\mathbf{O}_{\sigma_{S}}=: O_{S}$ for $S$ running over the set of vertices of $\mathcal{P}$. It also follows from the same arguments that the 1-dimensional orbits on $V^{\prime}$ contracted to $O$ are the $\mathbf{O}_{\sigma_{F}}$ for $F$ running over the set of compact 1-dimensional edges of $\mathcal{P}$. Hence the point $O_{S}$ lies on two irreducible components of the exceptional curve $E$ on $V^{\prime}$ if and only if $S$ lies in the relative interior of $\sigma^{\vee}$, i.e. $S \in \mathcal{S}$. Therefore, the map from $\mathcal{S}$ to the singular locus Sing $E$ of $E$ which sends $S$ to $O_{S}$ is a 1-1 correspondence.

Now, for $S \in \mathcal{S}, V^{\prime}$ has a rational double point of type $\mathbb{A}_{c_{S}-1}$ with $c_{S}=\frac{2 q_{S}}{p_{S}}$ (resp. is non singular) at $O_{S}$ if $\left(p_{S}, q_{S}\right) \neq(2,1)\left(\operatorname{resp} .\left(p_{S}, q_{S}\right)=(2,1)\right)$. To see that, it is enough to verify that, in a suitable basis of $N, \sigma_{S}=$ $\mathbb{R}_{\geq 0}(1,0)+\mathbb{R}_{\geq 0}\left(1, c_{S}\right)$. Recall that, by definition,

$$
\sigma_{S}=\left\{n \in N_{\mathbb{R}} \mid\left\langle S^{*}-S, n\right\rangle \geq 0 \text { for } *=+,-\right\}
$$

and that $S^{+}+S^{-}=\left(c_{S}+2\right) S$. Thus

$$
\sigma_{S}=\left\{n \in N_{\mathbb{R}} \mid c_{S}\langle S, n\rangle-\left\langle S^{+}-S, n\right\rangle \geq 0,\left\langle S^{+}-S, n\right\rangle \geq 0\right\}
$$

and the dual basis of the $\mathbb{Z}$-basis $\left(S, S^{+}-S\right)$ of $M$ enjoys the desired property. Note that the set $\left\{c_{S}\right\}_{S \in \mathcal{S}}$, or equivalently the set of pairs $\left\{\left(p_{S}, q_{S}\right)\right\}_{S \in \mathcal{S}}$, endowed with the order induced by the order on $\stackrel{\vee}{G}$ defined in lemma 3.9 , may be computed directly from the cone $\sigma$ attached to $V$ as follows: We may assume that $\sigma=\mathbb{R}_{>0}(1,0)+\mathbb{R}_{>0}(p, q)$ where $p, q$ are relatively prime integers satisfying $0<p<q$. Consider the continued fraction expansion

$$
\frac{q}{p}=\widetilde{c}_{1}-\frac{1}{\widetilde{c}_{2}-\frac{1}{\ddots \cdot-\frac{1}{\widetilde{c}_{e}}}}
$$

with $\widetilde{c}_{i} \geq 2$ for $1 \leq i \leq e$. Then, the $\left\{c_{S}\right\}$ is the sequence obtained by removing the zeros in the sequence $\left\{\widetilde{c}_{i}-2\right\}_{1 \leq i \leq e}$ by a result of Riemenschneider (see [O, lemma 1.22, corollary 1.23]).

The Nash blowing-up of $V$ has played a crucial rôle in the analysis of $\left[j^{s}(H)\right]$ in section 3. Here is another consequence of lemma 3.9 that we have not yet written down explicitly: Let $\widehat{V}$ denote the normal surface which dominates minimally the blowing-up $V^{\prime}$ of $V$ with center $O$ and its Nash blowing-up, as in lemma 3.9. For every $S \in \mathcal{S}$, the inverse image of 
$O_{S} \in V^{\prime}$ on $\widehat{V}$ is an irreducible curve $\widehat{E}_{S}=\overline{O_{\sigma_{\left[S^{-} S^{+}\right]}}}$and no other curve on $\widehat{V}$ than the $\widehat{E}_{S}, S \in \mathcal{S}$, is contracted to a point of $V^{\prime}$. In particular, Sing $E$ coincides with the set of base points of $\mathcal{J}_{l} \mathcal{O}_{V^{\prime}}$ (i.e. the points of $V^{\prime}$ at which $\mathcal{J}_{l} \mathcal{O}_{V^{\prime}}$ is not invertible). We will now show that the integers $q_{S}$, $q_{S}+p_{S}$ are the vanishing orders of suitable ideal sheaves on $\widehat{V}$ along $\widehat{E}_{S}$. We have seen in (4.1) that the isomorphism $\varphi: N \longrightarrow \mathbb{Z} \times \mathbb{Z}$ sending $n$ to $\left(\left\langle S^{*}-S, n\right\rangle,\left\langle S^{*}, n\right\rangle\right)$ maps $\sigma_{\left[S^{-} S^{+}\right]}$to $\mathbb{R}_{\geq 0}\left(q_{S}, q_{S}+p_{S}\right)$. This means that the primitive vector $\nu_{S}$ on $\sigma_{\left[S^{-} S^{+}\right]}$satisfies the linear system

$$
q_{S}=\left\langle S^{*}-S, \nu_{S}\right\rangle, \quad q_{S}+p_{S}=\left\langle S^{*}, \nu_{S}\right\rangle .
$$

Now, for any $m \in M$, the order of the zero or of the pole of $X^{m}$ along $\widehat{E}_{S}$ is $\left\langle m, \nu_{S}\right\rangle$. Since by $(3.7)$ and lemmas 3.8 and $3.9, \mathcal{M O}_{\widehat{V}}\left(\operatorname{resp} . \mathcal{J}_{l} \cdot \mathcal{M}^{-1} \mathcal{O}_{\widehat{V}}\right)$ is generated by $X^{S}$ (resp. $X^{S^{*}}$ ) on the affine open set $\widehat{V}_{\sigma_{S^{*}}}$ of $\widehat{V}$, which contains $\widehat{E}_{S} \backslash\left(O_{S^{+}} \cup O_{S^{-}}\right)$, we have

$$
\begin{aligned}
\left\langle S, \nu_{S}\right\rangle & =\operatorname{ord}_{\widehat{E}_{S}} \mathcal{M O}_{\widehat{V}}=p_{S} \\
\left\langle S^{*}, \nu_{S}\right\rangle & =\operatorname{ord}_{\widehat{E}_{S}} \mathcal{J}_{l} \mathcal{O}_{\widehat{V}}-\operatorname{ord}_{\widehat{E}} \mathcal{M O}_{\widehat{V}}=q_{S}+p_{S} \\
\left\langle S^{*}+S, \nu_{S}\right\rangle & =\operatorname{ord}_{\widehat{E}_{S}} \mathcal{J}_{l} \mathcal{O}_{\widehat{V}}=q_{S}+2 p_{S} \\
\left\langle S^{*}-S, \nu_{S}\right\rangle & =2\left(\operatorname{ord}_{\widehat{E}_{S}} \mathcal{J}_{l} \mathcal{O}_{\widehat{V}}-\operatorname{ord}_{\widehat{E}_{S}} \mathcal{M} \mathcal{O}_{\widehat{V}}\right)-\operatorname{ord}_{\widehat{E}_{S}} \mathcal{J}_{l} \mathcal{O}_{\widehat{V}}=q_{S}
\end{aligned}
$$

Also note that $h \in H_{\nu_{S}}^{*}$ if and only if its strict transform on $\widehat{V}$ is smooth and intersects transversally $\widehat{E}_{S}$ at a point distinct from $O_{S^{+}}$and $O_{S^{-}}$, and that $q_{S}+p_{S}$ is the minimal level of stability of $H_{\nu_{S}}^{*}$.

We now bring together and summarize the results in section 4 .

Theorem 4.5 Let $V$ be an affine normal toric surface with a 0 -dimensional closed orbit $O$, and let $H$ denote the set of arcs on $V$ going through $O$. Let $N$ be the lattice of one-parameter subgroups on the torus $T$, and let $\sigma$ be the 2-dimensional strongly convex cone in $N_{\mathbb{R}}:=N \otimes_{\mathbb{Z}} \mathbb{R}$ generated by the one-parameter subgroups on $T$ having a limit in $V$ for $t \rightarrow 0$.

Choose a $\mathbb{Z}$-basis $\epsilon_{1}, \epsilon_{2}$ of $N$ such that $\sigma=\mathbb{R}_{\geq 0} \epsilon_{1}+\mathbb{R}_{\geq 0}\left(p \epsilon_{1}+q \epsilon_{2}\right)$ for relatively prime integers $p, q$ with $0 \leq p<q$, and, if $p \neq 0$, write the continued fraction expansion

$$
\frac{q}{p}=\widetilde{c}_{1}-\frac{1}{\widetilde{c}_{2}-\frac{1}{\ddots-\frac{1}{\widetilde{c}_{e}}}}
$$


with $\widetilde{c}_{i} \geq 2,1 \leq i \leq e$. Denote by $\mathcal{S}$ the set of those $i, 1 \leq i \leq e$, such that $c_{i}:=\widetilde{c}_{i}-2 \neq 0$ (resp. $\emptyset$ ) for $p \neq 0$ (resp. $p=0$ ) and, for each $i \in \mathcal{S}$, let $p_{i}, q_{i}$ be relatively prime integers such that $c_{i} / 2=q_{i} / p_{i}$. Then

$$
\begin{aligned}
P_{\text {geom }}(T) & :=\sum_{s \geq 0}\left[j^{s}(H)\right] T^{s}= \\
& =\frac{1}{1-\mathbb{L}^{2} T}+(\mathbb{L}-1) \sum_{i \in \mathcal{S}} \frac{\stackrel{\circ}{Q}_{i}-\left(\left\lceil\frac{q_{i}}{p_{i}}\right\rceil-\left\lfloor\frac{q_{i}}{p_{i}}\right\rfloor\right) \mathbb{L}^{\left\lfloor\frac{q_{i}}{p_{i}}\right\rfloor} T^{\left\lceil\frac{q_{i}}{p_{i}}\right\rceil}}{\left(1-\mathbb{L}^{2} T\right)\left(1-\mathbb{L}^{q_{i}} T^{q_{i}+p_{i}}\right)}
\end{aligned}
$$

where

$$
\stackrel{\circ}{Q}_{i}:=\sum_{(a, b) \in \circ_{\Xi} \cap \cap \mathbb{Z}^{2}} \mathbb{L}^{a} T^{b} \quad \text { with } \quad \stackrel{\circ}{\Xi}_{i}=\left\{\lambda_{1}\left(q_{i}, q_{i}+p_{i}\right)+\lambda_{2}(1,0) \mid 0<\lambda_{i}<1\right\}
$$

Let $\pi^{\prime}: V^{\prime} \longrightarrow V$ be the blowing-up with center $O$, and let $E=\pi^{-1}(O)$ be the exceptional curve on $V^{\prime}$. Then, there is a one to one correspondence $i \mapsto O_{i}$ from $\mathcal{S}$ to Sing $E$ such that $V^{\prime}$ has a rational double point of type $\mathbb{A}_{c_{i}-1}$ (resp. is non singular) at $O_{i}$ for $\left(p_{i}, q_{i}\right) \neq(2,1)$ (resp. $=(2,1)$ ).

Let $\mathcal{J}_{l}$ denote the logarithmic-jacobian ideal in $\mathbf{k}[V]$ (see appendix), and let $\widehat{\pi}: \widehat{V} \longrightarrow V^{\prime}$ be the blowing-up with center $\mathcal{J}_{l} \mathcal{O}_{V^{\prime}}$ followed by normalization. Then Sing $E$ coincides with the set of base points of $\mathcal{J}_{l} \mathcal{O}_{V^{\prime}}$. Moreover, for each $i \in \mathcal{S}, \widehat{\pi}^{-1}\left(O_{i}\right)$ is an irreducible curve $\widehat{E}_{i} \cong \mathbb{P}_{\mathbf{k}}^{1}$, and we have

$$
\operatorname{ord}_{\widehat{E}_{i}} \mathcal{M O}_{\widehat{V}}=p_{i}, \quad \nu_{i}:=\operatorname{ord}_{\widehat{E}_{i}} \mathcal{J}_{l} \mathcal{O}_{\widehat{V}}=q_{i}+2 p_{i}
$$

Remark: The above formula for $P_{\text {geom }}(T)$ holds in $K_{0}\left(\mathcal{V}_{\mathbf{k}}\right)[[T]]$.

Corollary 4.6 For normal toric surface singularities as above, $P_{\text {geom }}(T)$ has only simple poles.

Corollary 4.7 Assume $\mathbf{k}=\mathbb{C}$ and let $\overline{\mathcal{M}_{\mathbf{k}}}$ denote the image of $\mathcal{M}_{\mathbf{k}}$ in the Kontsevich's completion $\widehat{\mathcal{M}_{\mathbf{k}}}$. Let $\chi: \overline{\mathcal{M}_{\mathbf{k}}}\left[\left(\frac{\mathbb{L}-1}{\mathbb{L}^{j}-1}\right)_{j \geq 1}\right] \longrightarrow \mathbb{Q}$ be the extension of the topological Euler characteristic (see [D-L1] sections 1 and 6). In the notation of theorem 4.5, we have

$$
\chi(\mu(H))=1+\sum_{i \in \mathcal{S}} \frac{q_{i}}{q_{i}+2 p_{i}}
$$

Proof: Recall that, by definition, $\chi\left(\frac{\mathbb{L}-1}{\mathbb{L}^{j}-1}\right)=\frac{1}{j}$. The assertion follows immediately from formula (4.3.5) for the motivic volume. 
Corollary 4.8 Let $V^{(j)}, j=1,2$, be affine normal toric surfaces with 0 -dimensional closed orbits and let $P_{\text {geom }}^{(j)}(T), j=1,2$ be the corresponding series. The following conditions are equivalent:

i) $P_{\text {geom }}^{(1)}(T)=P_{\text {geom }}^{(2)}(T)$.

ii) Let $\mathcal{S}^{(j)} \longrightarrow \mathbb{Z}_{\geq 1}, i \mapsto c_{i}^{(j)}, j=1,2$, be the sequence of positive integers obtained from the continued fraction expansion process as explained in theorem 4.5. There exists a bijective map $\omega: \mathcal{S}^{(1)} \longrightarrow \mathcal{S}^{(2)}$ such that $c_{i}^{(1)}=c_{\omega(i)}^{(2)}$ for any $i \in \mathcal{S}^{(1)}$.

iii) Let $V^{(j)^{\prime}} \longrightarrow V^{(j)}$ be the blowing-up with center the maximal ideal $\mathcal{M}^{(j)}$ defining the closed orbit on $V^{(j)}$, and let $E^{(j)}$ denote the exceptional curve on $V^{(j)^{\prime}}, j=1,2$. There exists a bijective map from the singular locus of $E^{(1)}$ to the singular locus of $E^{(2)}, O_{i}^{(1)} \mapsto O_{\omega(i)}^{(2)}$, such that, for any $O_{i}^{(1)} \in \operatorname{Sing} E^{(1)}$, the germs $\left(V^{(1)^{\prime}}, O_{i}^{(1)}\right)$ and $\left(V^{(2)^{\prime}}, O_{\omega(i)}^{(2)}\right)$ are analytically isomorphic.

iv) Let $\mathcal{J}_{l}^{(j)}$ denote the logarithmic-jacobian ideal on $V^{(j)}$, and let $\widehat{E}^{(j)}$ denote the exceptional curve of the blowing-up $\widehat{V^{(j)}} \longrightarrow V^{(j)^{\prime}}$ with center $\mathcal{J}_{l}^{(j)} \mathcal{O}_{V^{(j)^{\prime}}}, j=1,2$. There exists a bijective map from the set of irreducible components of $\widehat{E}^{(1)}$ to the set of irreducible components of $\widehat{E}^{(2)}$, $\widehat{E}_{i}^{(1)} \mapsto \widehat{E}_{\omega(i)}^{(2)}$, such that, for any irreducible component $\widehat{E}_{i}^{(1)}$ of $\widehat{E}^{(1)}$,

$$
\operatorname{ord}_{\widehat{E}_{i}^{(1)}}\left(\mathcal{M}^{(1)} \mathcal{O}_{\widehat{V^{(1)}}}\right)=\operatorname{ord}_{\widehat{E}_{\omega(i)}^{(2)}}\left(\mathcal{M}^{(2)} \mathcal{O}_{\widehat{V^{(2)}}}\right)
$$

and

$$
\operatorname{ord}_{\widehat{E}_{i}^{(1)}}\left(\mathcal{J}_{l}^{(1)} \mathcal{O}_{\widehat{V^{(2)}}}\right)=\operatorname{ord}_{\widehat{E}_{\omega(i)}^{(1)}}\left(\mathcal{J}_{l}^{(1)} \mathcal{O}_{\widehat{V^{(1)}}}\right)
$$

Proof: The equivalences $i i) \Longleftrightarrow i i i) \Longleftrightarrow i v$ ) follow from the discussion in (4.4) and they imply $i$ ) by theorem 4.5 .

Now suppose that $i$ ) holds. The poles of $P_{\text {geom }}^{(j)}(T)$ are in 1-1 correspondence with the image of $\mathcal{S}^{(j)}$ in $\mathbb{Z}_{\geq 1}$, namely the set $\left\{c_{i}^{(j)} \mid i \in \mathcal{S}^{(j)}\right\}$. Let $B_{i}=1-\mathbb{L}^{q_{i}} T^{q_{i}+p_{i}}, 1 \leq i \leq \tau$, be the poles of $P=P_{\text {geom }}^{(j)}(T), j=1,2$. Since every $B_{i}$ is irreducible, there is only one way to write $\left[\left(1-\mathbb{L}^{2} T\right) P-1\right](\mathbb{L}-$ $1)^{-1}$ as a sum $\sum_{1 \leq i \leq \tau} A_{i} / B_{i}$ with $A_{i} \in \mathbb{Z}[\mathbb{L}, T]$ of degree $<p_{i}+q_{i}$ with respect to $T$. Hence the multiplicity of $c_{i}=2 q_{i} / p_{i}$ in the image of $\mathcal{S}^{(j)}$, $j=1,2$, is $A_{i}(1,1) / q_{i}$ and $\left.i i\right)$ holds. 
Here is another immediate consequence of theorem 4.5.

Corollary 4.9 In the notation of theorem 4.5, the following conditions are equivalent:

i) $P_{\text {geom }}(T)=\frac{1}{1-\mathbb{L}^{2} T}$.

ii) There exists an integer $q \geq 1$ and a $\mathbb{Z}$-basis $\epsilon_{1}, \epsilon_{2}$ of $N$ such that $\sigma=\mathbb{R}_{\geq 0} \epsilon_{1}+\mathbb{R}_{\geq 0}\left((q-1) \epsilon_{1}+q \epsilon_{2}\right)$.

iii) The exceptional curve $E$ of $V^{\prime}$ is irreducible (equivalently nonsingular).

iv) $\mathcal{J}_{l} \mathcal{O}_{V^{\prime}}$ has no base points.

If these conditions hold, then $V^{\prime}$ is nonsingular and $-E^{2}$ (or equivalently, the multiplicity of $V$ at $O$ ) is the integer $q$ of ii).

Therefore, toric surface singularities may share the same $P_{\text {geom }}(T)$ without being analytically isomorphic. Here is another example.

Example: Let $\left(\epsilon_{1}, \epsilon_{2}\right)$ be the canonical basis of $N \cong \mathbb{Z}^{2}$, and let $\sigma_{1}=$ $\mathbb{R}_{\geq 0} \epsilon_{1}+\mathbb{R}_{\geq 0}\left(\epsilon_{1}+3 \epsilon_{2}\right)$. The continued fraction expansion of $3 / 1$ as in theorem 4.5 is reduced to $\widetilde{c}_{1}=3$, hence $c_{1}=1, p_{1}=2$ and $q_{1}=1$. Let now $\sigma_{2}=\mathbb{R}_{\geq 0} \epsilon_{1}+\mathbb{R}_{\geq 0}\left(3 \epsilon_{1}+5 \epsilon_{2}\right)$. The continued fraction expansion of $5 / 3$ is $5 / 3=2-1 / 3$. Here we have $\widetilde{c}_{1}=2, \widetilde{c}_{2}=3$, hence $c_{1}=0$ and $c_{2}=1$.

By theorem 4.5, we have

$$
P_{\text {geom }}(T)=\frac{1}{1-\mathbb{L}^{2} T}+(\mathbb{L}-1) \frac{\left[(\mathbb{L}-1) T+\mathbb{L} T^{2}\right]}{\left(1-\mathbb{L}^{2} T\right)\left(1-\mathbb{L} T^{3}\right)}
$$

for the surface $V^{(1)}$ given by $\sigma_{1}$ as well as for $V^{(2)}$ given by $\sigma_{2}$. The minimal desingularization of both surfaces is obtained by blowing up the singular point, and the exceptional curve consists of 2 projective lines intersecting at a base point of the inverse image of the logarithmic-jacobian. The singularity of $V^{(1)}$ is a rational double point of type $\mathbb{A}_{2}$, whose weighted dual graph is shown in Fig. 1. The singularity of $V^{(2)}$ is a rational triple point whose weighted dual graph is shown in Fig. 2.

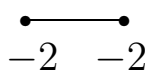

FIG. 1

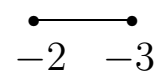

FIG. 2

Remark: These examples show that neither the weighted dual graph nor the multiplicity of a normal surface singularity can be read off $P_{\text {geom }}(T)$. However, the argument in the proof of corollary 4.8 shows that, if $O$ is a singular point of a normal toric surface singularity, one can recover the number of irreducible exceptional curves on its minimal desingularization (or equivalently here, the dual graph) from $P_{\text {geom }}(T)$. 
(4.10) Theorem 4.5 and its corollaries suggest several questions:

Question 1: The singularities of toric varieties are rational singularities (see [TE, chap. I.3], [O, cor. 3.9]). Following Abhyankar, a surface singularity is called quasirational if "only rational curves can come out of the singularity of the surface, no matter how we blow it up birationally" ([A, p. 267]). A rational surface singularity is quasirational. Is it true that, for any rational (resp. quasirational) surface singularity, the series $P_{\text {geom }}(T)$ is a rational function with numerator (and denominator) in $\mathbb{Z}[\mathbb{L}][T]$ ? If the answer is yes, does this property characterize rational (resp. quasirational) surface singularities?

Question 2: The series $P_{\text {geom }}(T)$, hence the motivic volume $\mu(H)$, for a toric surface singularity $(V, O)$ only depend on the blowing-up with center $O$ and the Nash blowing-up of $V$. This shows in particular that not every exceptional irreducible curve on a desingularization of $V$ dominating its Nash blowing-up as in [D-L1, proposition 6.3.2], gives rise to an actual pole of $\mu(H)$. Does the same hold for normal surface singularities, for isolated singularities of normal algebraic varieties of any dimension?

Question 3: Denef and Loeser have introduced another series $P_{\mathrm{ar}}(T)$ in [D-L2]. Is it true that $P_{\text {geom }}(T)=P_{\text {ar }}(T)$ for toric surface singularities? It would also be interesting to compare these series with $J(T)=\sum_{s>0}\left[H_{s}\right] T^{s}$ where $H_{s}$ is the set of $\mathbf{k}$-local homomorphisms $\mathcal{O}_{V, O} \longrightarrow \mathbf{k}[[t]] /(t)^{s+1}$ and $\left[H_{s}\right]$ denotes the image of its reduced underlying variety in $\mathcal{M}_{\mathbf{k}}$.

Question 4: Compute $P_{\text {geom }}(T)$ for a normal toric variety of dimension $\geq 3$.

\section{A. Appendix. Toric varieties and Nash blowing-up}

Let $V$ be an affine normal toric variety over an algebraically closed field $\mathbf{k}$ of characteristic zero. González-Sprinberg has exhibited an ideal of its ring of regular functions $\mathbf{k}[V]$ whose blowing-up is the Nash blowing-up of $V$ ([GS1]). We give an alternative proof of his result using differential forms with logarithmic poles along the lines of [P, section 4].

Let $X$ be an algebraic variety, reduced and irreducible over the algebraically closed field $\mathbf{k}$ of characteristic zero. Recall that a proper birational map $\pi: \widetilde{X} \longrightarrow X$ is called a Nash blowing-up of $X$ if $\pi^{*} \Omega_{X}^{1} /$ torsion is a locally free $\mathcal{O}_{\tilde{X}}$-module and if it has the following universal property: if $\pi^{\prime}: \widetilde{X}^{\prime} \longrightarrow X$ is a proper birational map such that $\pi^{\prime *} \Omega_{X}^{1} /$ torsion is a locally free $\mathcal{O}_{\widetilde{X}^{\prime}}$-module, then there exists a unique morphism $\widetilde{\pi}: \widetilde{X}^{\prime} \longrightarrow \widetilde{X}$ with $\pi \circ \tilde{\pi}=\pi^{\prime}$. Here $\Omega_{X}^{1}$ is the sheaf of Kähler differentials of $X$ over $\mathbf{k}$. The universal property characterizes the Nash blowing-up of $X$ up to isomorphism. 
On our affine toric variety $V$ as above, there exists a distinguished $T$-stable Weil divisor $D$, namely the sum of all codimension one $T$-orbit closures. Let $M$ denote the lattice of exponents of monomials on the torus $T \cong\left(\mathbf{k}^{*}\right)^{d}$ inside $V$. The Kähler module of differentials $\Omega_{\mathbf{k}[T] / \mathbf{k}}^{1}$ is canonically isomorphic to $\mathbf{k}[T] \otimes_{\mathbb{Z}} M$ via the map $\frac{d x^{m}}{x^{m}} \mapsto 1 \otimes m$, for any $m \in M$. For $p=0, \ldots, d$, the sheaf of germs of $p$-forms with logarithmic poles along $D$, denoted $\Omega_{V}^{p}(\log \mathrm{D})$, is canonically isomorphic to $\mathcal{O}_{V} \otimes_{\mathbb{Z}} \wedge^{p} M$, and there exists a canonical $\mathcal{O}_{V}$-homomorphism

$$
\Omega_{V}^{p} \longrightarrow \Omega_{V}^{p}(\log D)=\mathcal{O}_{V} \otimes_{\mathbb{Z}} \wedge^{p} M
$$

which maps $X^{m} d X^{m_{1}} \wedge \ldots \wedge d X^{m_{p}}$ to $X^{m+m_{1}+\ldots+m_{p}} \otimes\left(m_{1} \wedge \ldots \wedge m_{p}\right)$. Here $X^{m}, X^{m_{1}}, \ldots, X^{m_{p}}$ are global sections of $\mathcal{O}_{V}$, or equivalently $m, m_{1}, \ldots, m_{p} \in$ $\sigma^{\vee} \cap M$ with $V=V_{\sigma}$ (see [O, chapter 3]).

We define the logarithmic-jacobian ideal $\mathcal{J}_{l}$ of $V$ to be the image of the Kähler module of differentials $\Omega_{\mathbf{k}[V] / \mathbf{k}}^{d}$ in $\mathbf{k}[V]$, identified with $\mathbf{k}[V] \otimes_{\mathbb{Z}} \wedge^{d} M$. In view of $(1), \mathcal{J}_{l}$ is the ideal generated by $X^{\mu_{i_{1}}+\ldots+\mu_{i_{d}},} i_{1}<\ldots<i_{d}$, where $\mu_{i_{1}}, \ldots, \mu_{i_{d}}$ is running over the set of $d$-uples of $\mathbb{R}$-linearly independent elements in the minimal generating system $\stackrel{\vee}{G}=\left(\mu_{i}\right)_{i \in I}$ of $\sigma^{\vee} \cap M$. This is precisely the ideal introduced in [GS1, section 2].

Proposition A.1 Let $V$ be an affine normal toric variety over an algebraically closed field $\mathbf{k}$ of characteristic zero. The blowing-up of $V$ with center its logarithmic-jacobian is the Nash blowing-up of $V$.

The proof of the proposition uses the following observation:

Lemma A.2 Let $p$ be a positive integer. Let $A$ be an integral domain with quotient field $K$, let $M$ be an $A$-module and let $\varphi: M \longrightarrow A^{p}$ be an A-linear map such that $\varphi \otimes 1: M \otimes_{A} K \longrightarrow K^{p}$ is an isomorphism. Then $\varphi$ induces an isomorphism from $M /$ torsion to the image of $\varphi$.

Proof: The claim is equivalent to saying that the torsion submodule of $M$, namely the set of those $m \in M$ with a nonzero annihilator $f \in A$ is the kernel of $\varphi$, which is obvious.

Proof of the proposition: Let $\pi: \widetilde{V} \longrightarrow V$ denote the blowing-up with center $\mathcal{J}_{l}$. It follows from the above lemma applied locally at each point of $\widetilde{V}$ that $\pi^{*} \Omega_{V}^{1}$ /torsion is isomorphic to the image $\widetilde{\Omega}^{1}$ of $\pi^{*} \Omega_{V}^{1}$ by the canonical map

$$
\pi^{*} \Omega_{V}^{1} \longrightarrow \pi^{*} \Omega_{V}^{1}(\log D)=\mathcal{O}_{\widetilde{V}} \otimes_{\mathbb{Z}} M
$$

induced by (1). 
Let $\widetilde{V}_{i_{1}, \ldots, i_{d}}$ denote the affine open set of $\widetilde{V}$ on which the restriction of $\mathcal{J}_{l} \mathcal{O}_{\widetilde{V}}$ is generated by $X^{\mu_{i_{1}}+\ldots+\mu_{i_{d}}}$. Since $\mu_{i_{1}}, \ldots, \mu_{i_{d}}$ are $\mathbb{R}$-linearly independent elements in $\mathbb{Z}^{d}$, for any $\mu \in \mathscr{V}$, there exist $a_{i_{j}} \in \mathbb{Z}, 1 \leq j \leq d$, such that

$$
\operatorname{det}\left(\mu_{i_{1}}, \ldots, \mu_{i_{d}}\right) \mu=\sum_{j=1}^{d} a_{i_{j}} \mu_{i_{j}}
$$

Moreover, since $a_{i_{j}}$ is equal to $\mu \wedge \mu_{i_{1}} \wedge \ldots \wedge \widehat{\mu_{i_{j}}} \wedge \ldots \wedge \mu_{i_{d}}$ up to sign, we have $a_{i_{j}} \neq 0$ if and only if $\mu, \mu_{i_{1}}, \ldots, \widehat{\mu_{i_{j}}}, \ldots, \mu_{i_{d}}$ are $\mathbb{R}$-linearly independent. Here $\widehat{\mu_{i_{j}}}$ means that $\mu_{i_{j}}$ is omitted in the list.

By definition, the image of $d X^{\mu}$ is $X^{\mu} \otimes \mu$. Since $\delta:=\operatorname{det}\left(\mu_{i_{1}}, \ldots, \mu_{i_{d}}\right)$ is a nonzero integer, and $\mathbf{k}$ is a field of characteristic zero, we have

$$
\begin{aligned}
X^{\mu} \otimes \mu & =\quad \frac{1}{\delta} X^{\mu} \otimes \delta \mu=\frac{1}{\delta} X^{\mu} \otimes \sum_{j} a_{i_{j}} \mu_{i_{j}} \\
& =\sum_{j} \frac{a_{i_{j}}}{\delta} X^{\mu} \otimes \mu_{i_{j}}=\sum_{j} \frac{a_{i_{j}}}{\delta} X^{\mu-\mu_{i_{j}}}\left(X^{\mu_{i_{j}}} \otimes \mu_{i_{j}}\right)
\end{aligned}
$$

Now, if $a_{i_{j}} \neq 0$, the monomial $X^{\mu+\mu_{i_{1}}+\ldots+\widehat{\mu_{i_{j}}}+\ldots+\mu_{i_{d}}}$ belongs to $\mathcal{J}_{l}$. This monomial is equal to $X^{\mu-\mu_{i_{j}}} X^{\mu_{i_{1}}+\ldots+\mu_{i_{d}}}$, therefore $X^{\mu-\mu_{i_{j}}}$ is a section of $\mathcal{O}_{\widetilde{V}}$ on $\widetilde{V}_{i_{1}, \ldots, i_{d}}$. This shows that, for any $x \in \widetilde{V}_{i_{1}, \ldots, i_{d}}$ (closed or not), the stalk $\widetilde{\Omega}_{x}^{1}$ is the image of the $\mathcal{O}_{\tilde{V}, x}$-homomorphism

$$
\varphi_{i_{1}, \ldots, i_{d}}: \mathcal{O}_{\widetilde{V}, x} \otimes_{\mathbb{Z}} M \longrightarrow \mathcal{O}_{\widetilde{V}, x} \otimes_{\mathbb{Z}} M
$$

which maps $1 \otimes e_{j}$ to $X^{\mu_{i_{j}}} \otimes \mu_{i_{j}}, 1 \leq j \leq d$. Here $e_{1}, \ldots, e_{d}$ is the canonical basis of $M \cong \mathbb{Z}^{d}$.

Taking the $d$-th exterior power, we get a commutative diagram

By definition, we have

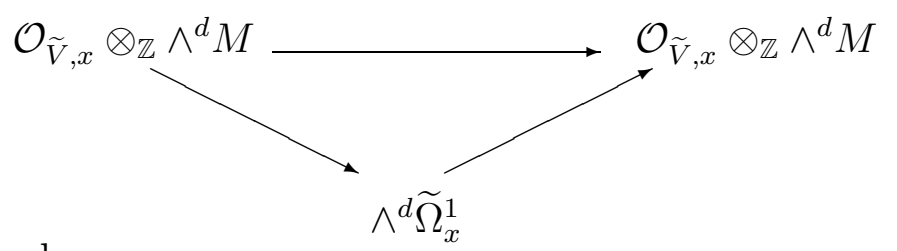

$$
\wedge^{d} \varphi_{i_{1}, \ldots, i_{d}}\left(1 \otimes\left(e_{1} \wedge \ldots \wedge e_{d}\right)\right)=X^{\mu_{i_{1}}+\ldots+\mu_{i_{d}}} \otimes\left(\mu_{i_{1}} \wedge \ldots \wedge \mu_{i_{d}}\right)
$$

so $\wedge^{d} \varphi_{i_{1}, \ldots, i_{d}}$ induces an isomorphism of $\mathcal{O}_{\widetilde{V}, x} \otimes_{\mathbb{Z}} \wedge^{d} M$ with its image

$$
\mathcal{J}_{l} \mathcal{O}_{\widetilde{V}, x} \otimes_{\mathbb{Z}} \wedge^{d} M
$$

In addition, this shows that the map $\mathcal{O}_{\widetilde{V}, x} \otimes_{\mathbb{Z}} \wedge^{d} M \longrightarrow \wedge^{d} \widetilde{\Omega}_{x}^{1}$ is injective. Since it is also surjective, we conclude that $\wedge^{d} \widetilde{\Omega}^{1}$ is an invertible $\mathcal{O}_{\widetilde{V}^{-}}$-module canonically isomorphic to $\mathcal{J}_{l} \mathcal{O}_{\widetilde{V}} \otimes_{\mathbb{Z}} \wedge^{d} M$. 
This implies that $\widetilde{\Omega}^{1}$ is a locally free $\mathcal{O}_{\widetilde{V}}$-module of rank $d$. Indeed, for any $x \in \widetilde{V}$, we have $\operatorname{dim}_{\mathbf{k}(x)} \wedge^{d}\left(\widetilde{\Omega}_{x}^{1} \otimes \mathbf{k}(x)\right)=\operatorname{dim}_{\mathbf{k}(x)}\left(\wedge^{d} \widetilde{\Omega}_{x}^{1}\right) \otimes \mathbf{k}(x)=1$, where $\mathbf{k}(x)$ denotes the residue field of $\widetilde{V}$ at $x$. Therefore, for any $x \in \widetilde{V}$, we have $\operatorname{dim}_{\mathbf{k}(x)}\left(\widetilde{\Omega}_{x}^{1} \otimes \mathbf{k}(x)\right)=d$ which is known to be equivalent to the claim.

Also note that

$$
\wedge^{d}\left(\pi^{*} \Omega_{V}^{1} / \text { torsion }\right)=\pi^{*} \Omega_{V}^{d} / \text { torsion }
$$

Indeed, we already know that the $\mathcal{O}_{\widetilde{V}}$-modules $\wedge^{d}\left(\pi^{*} \Omega_{V}^{1} /\right.$ torsion $), \wedge^{d} \widetilde{\Omega}^{1}$ and $\mathcal{J}_{l} \mathcal{O}_{\widetilde{V}} \otimes \wedge^{d} M$ are canonically isomorphic. Now recall that, by definition, $\mathcal{J}_{l} \otimes_{\mathbb{Z}} \wedge^{d} M$ is the image of the map $\Omega_{V}^{d} \longrightarrow \mathcal{O}_{V} \otimes_{\mathbb{Z}} \wedge^{d} M$ in (1). Therefore $\mathcal{J}_{l} \mathcal{O}_{\widetilde{V}} \otimes \wedge^{d} M$ is the image of the induced map $\pi^{*} \Omega_{V}^{d} \longrightarrow \mathcal{O}_{\widetilde{V}} \otimes_{\mathbb{Z}} \wedge^{d} M$. But, by lemma A.2 again, this map induces an isomorphism of $\pi^{*} \Omega_{V}^{d} /$ torsion with its image $\mathcal{J}_{l} \mathcal{O}_{\widetilde{V}} \otimes \wedge^{d} M$. The canonical isomorphism between the modules in (2) thus obtained coincides with the identity over the inverse image of the regular locus of $V$, hence equality (2).

It only remains to prove that $\pi$ satisfies the universal property. Suppose that $\varphi: W \longrightarrow V$ is a proper birational map such that $\varphi^{*} \Omega_{V}^{1} /$ torsion is locally free. Since $\Omega_{V}^{1}$ is generically locally free of rank $d$, our hypothesis implies that $\wedge^{d}\left(\varphi^{*} \Omega_{V}^{1} /\right.$ torsion $)$ is invertible. Now the map

$$
\varphi^{*} \Omega_{V}^{d} \longrightarrow \wedge^{d}\left(\varphi^{*} \Omega_{V}^{1} / \text { torsion }\right)
$$

is surjective, so we deduce from lemma A.2 that $\varphi^{*} \Omega_{V}^{d} /$ torsion is invertible.

On the other hand, $\mathcal{J}_{l} \otimes_{\mathbb{Z}} \wedge^{d} M$ is the image of the map $\Omega_{V}^{d} \longrightarrow \mathcal{O}_{V} \otimes_{\mathbb{Z}}$ $\wedge^{d} M$, thus $\mathcal{J}_{l} \mathcal{O}_{W} \otimes_{\mathbb{Z}} \wedge^{d} M$ is the image of the map $\varphi^{*} \Omega_{V}^{d} \longrightarrow \mathcal{O}_{W} \otimes_{\mathbb{Z}} \wedge^{d} M$, hence this module is canonically isomorphic to $\varphi^{*} \Omega_{V}^{d} /$ torsion. This shows that $\mathcal{J}_{l} \mathcal{O}_{W}$ is invertible and we get our factorization by the universal property of blowing-up.

\section{References}

[A] Abhyankar, S. S.: Quasirational singularities. Amer. J. Math. 101 (1979), no. 2, 267-300.

[D-L1] Denef, J. And Loeser, F.: Germs of arcs on singular algebraic varieties and motivic integration. Invent. Math. 135 (1999), 201-232.

[D-L2] Denef, J. And Loeser, F.: Definable sets, motives and $p$-adic integrals. J. Amer. Math. Soc. 14 (2001), no. 2, 429-469.

[D-L3] Denef, J. And Loeser, F.: Geometry on arc spaces of algebraic varieties. In European Congress of Mathematics, Vol. I (Barcelona, 2000), 327-348. Progr. Math. 201, Birkhäuser, Basel, 2001. 
[GS1] GonzÁlez-Sprinberg, G.: Eventails en dimension 2 et transformé de Nash. Publications de l'E.N.S., Paris, 1977.

[GS2] GonzÁlez-Sprinberg, G.: Résolution de Nash des points doubles rationnels. Ann. Inst. Fourier (Grenoble) 32 (1982), no. 2, 111-178.

[GS3] GonzÁlez-SprinberG, G.: L'obstruction locale d'Euler et le théorème de Mac-Pherson. In The Euler-Poincaré characteristic, 7-32. Astérisque 82- 83, Soc. Math. France, Paris, 1981.

[L] Lipman, J.: Rational singularities with applications to algebraic surfaces and unique factorization. Inst. Hautes Études Sci. Publ. Math. 36 (1969), $195-279$.

[Lo] LooiJenga, E.: Motivic measures. Séminaire Bourbaki, 1999/2000. Astérisque 276 (2002), 267-297. Available on math. AG/0006220.

[L-T] Lê, D. T. AND Teissier, B.: Limites d'espaces tangents en géométrie analytique. Comment. Math. Helv. 63 (1988), 540-578.

[N] NAsh, J.: Arc structure of singularities. Duke Math. J. 81 (1995), 31-38.

[O] OdA, T.: Convex bodies and algebraic geometry . An introduction to the theory of toric varieties. Ergeb. Math. Grenzgeb. 15, Springer-Verlag, Berlin, 1988.

[P] Piene, R.: Ideals associated to a desingularization. In Algebraic Geometry (Proc. Summer Meeting, Univ. Copenhagen, Copenhagen, 1978), 503-517. Lecture Notes in Math 732, Springer-Verlag, Berlin, 1979.

[TE] Kempf, G., Knudsen, F., Mumford, D. and Saint-Donat, B.: Toroidal embeddings I. Lecture Notes in Math. 339, Springer-Verlag, 1973.

Recibido: 10 de abril de 2002

Monique Lejeune-Jalabert

CNRS, LAMA UMR 8100, CNRS-UVSQ

Bâtiment Fermat

45 Av. des Etas-Unis

F-78035 Versailles Cedex, France lejeune@math.uvsq.fr

Ana J. Reguera

Dpto. Álgebra y Geometría

Universidad de Valladolid

E-47005 Valladolid, Spain areguera@agt.uva.es

Dedicado al Profesor J.L. Vicente Córdoba en su 60 aniversario. Both authors are partially supported by Action Intégrée 02548 UB, Acción Integrada AI HF2000-076. 